University of Zurich

Department of Economics

Working Paper Series

ISSN 1664-7041 (print)

ISSN 1664-705X (online)

Working Paper No. 176

\title{
The Societal Benefit of a Financial Transaction Tax
}

Aleksander Berentsen, Samuel Huber and Alessandro Marchesiani

Revised version, July 2016 


\title{
The Societal Benefit of a Financial Transaction Tax*
}

\author{
Aleksander Berentsen \\ University of Basel and Federal Reserve Bank of St. Louis \\ Samuel Huber \\ Alessandro Marchesiani \\ University of Basel \\ University of Liverpool
}

July 16, 2016

\begin{abstract}
We provide a novel justification for a financial transaction tax for economies, where agents face stochastic consumption opportunities. A financial transaction tax makes it more costly for agents to readjust their portfolios of liquid and illiquid assets in response to these liquidity shocks, which increases the demand for - and the price of liquid assets. The higher price improves liquidity insurance and welfare for other market participants. We calibrate the model to U.S. data and find that the optimal financial transaction tax is 1.6 percent and that it reduces the volume of financial trading by 17 percent.
\end{abstract}

\section{Introduction}

A financial transaction tax (FTT) is a proportional tax on financial transactions. One of the early advocates was Tobin (1978) who proposed it in order to add some frictions into the "excessively efficient international money markets" (p. 154). Although Tobin's proposal was a proportional tax on currency transactions, the term "Tobin tax" is commonly used today for a proportional tax levied on any financial asset transaction.

The existing theoretical literature on FTTs focuses mainly on historical episodes or provides basic intuition in favor or against such a tax. Although this literature discusses many dynamic issues such as price volatility and liquidity in financial markets, the analysis is most often static. Furthermore, none of these papers studies the underlying frictions that give rise to the need for financial transactions in the first place, and the reader is left puzzled about what distortion a

${ }^{*}$ The views expressed in this article are those of the authors and not necessarily those of the Federal Reserve Bank of St. Louis, the Federal Reserve System, or the FOMC. Any remaining errors are the authors' responsibility. Aleksander Berentsen is a professor of economics at the Department of Economic Theory, University of Basel. Email: aleksander.berentsen@unibas.ch. Samuel Huber is a research fellow at the Department of Economic Theory, University of Basel. E-mail: samuel_h@gmx.ch. Alessandro Marchesiani is a senior lecturer of economics at the Management School, University of Liverpool. E-mail: marchesiani@gmail.com. 
FTT is intended to correct. Finally, very few studies offer a rigorous analysis of the welfare implications of FTTs.

Building on recent advances in monetary theory, we can now address these shortcomings by building a choice-theoretic dynamic general equilibrium model with frictions that make financial trading essential. ${ }^{1}$ The model allows us to address important positive and normative questions regarding the impact of a FTT on the real economy: For example, under which conditions is a FTT desirable and what distortion is corrected by such a tax? More generally, what is the optimal FTT and how does it affect trading volumes in financial markets?

In our model, agents face idiosyncratic random consumption and production opportunities and they hold a portfolio of liquid and illiquid assets. The liquid asset can be directly traded for consumption goods if a consumption opportunity arises; i.e., it serves as a medium of exchange. In contrast, the illiquid asset cannot be used as a medium of exchange. ${ }^{2}$ From the agents' point of view, the random consumption and production opportunities are liquidity shocks. These shocks generate an ex-post inefficient allocation of the medium of exchange: Some agents will hold liquid assets, but have no current need for them, while other agents will hold insufficient liquidity for their liquidity needs. To mitigate this liquidity mismatch, a financial market opens that allows the exchange of illiquid assets for liquid assets. The financial market is an over-the-counter (OTC) market, where agents are matched in pairs and the terms of trades are bargained.

Our main finding is that the portfolio choice of liquid and illiquid assets displays a pecuniary externality which results in an inefficiently low demand for the liquid asset. The reason for the pecuniary externality is that an agent does not take into account that, by holding more liquid assets, he not only acquires additional insurance against his own idiosyncratic liquidity risks, but he also marginally increases the value of the liquid asset, which improves the insurance for other market participants, too. This pecuniary externality can be corrected by a FTT. By making it more costly to readjust a portfolio in response to liquidity shocks, agents attempt to hold more of the liquid asset ex-ante. The resulting increase in the demand for liquid assets drives up the value of these assets, and this effect can be so strong that it is welfare-increasing. ${ }^{3}$

To provide a quantitative assessment for the optimal FTT, we calibrate the model to U.S. data. For the calibration, we assume that the FTT is zero. We then perform the following experiment: We search numerically for the tax rate that maximizes welfare. We find that for the United States, the optimal tax rate is 1.6 percent and that the optimal tax rate reduces the real volume of financial trading by 17 percent. As a robustness check, we also calibrate the model to Germany and find that the optimal tax rate is 1.5 percent.

The optimal rate of a FTT mainly depends on the financial market characteristics, which are captured in a OTC market by the matching probability and the bargaining power. For example, we find that the optimal tax rate decreases monotonically in the matching probability.

\footnotetext{
${ }^{1}$ These frictions include a lack of record-keeping (public communication of individual trading histories) and a lack of commitment. By essential, we mean that financial trading improves the allocation.

${ }^{2}$ It has been shown by Kocherlakota (2003) that an arrangement with illiquid bonds is efficient. See also Berentsen and Waller (2011) for a discussion on the societal benefits of illiquid bonds. We provide a short discussion of this result in Section 7 .

${ }^{3}$ The pecuniary externality arises in the steady state equilibrium.
} 
Furthermore, as the bargaining power of the agents who demand liquidity increases, the optimal tax rate increases.

FTT rates vary substantially and range from 0.1 percent in the European Union to 2 percent in the United States and Switzerland. The European Commission intends to introduce a FTT on the exchange of shares and bonds of 0.1 percent. The proposal is supported by eleven member states and is scheduled to be introduced in 2016. In the United States, the recent reform of the Securities and Exchange Commission, effective as of $14^{\text {th }}$ October 2014, allows money market funds to impose an exit fee of up to 2 percent. This so-called liquidity fee can be imposed if the fund's liquid assets fall below a pre-specified threshold. Similar regulatory changes have been imposed by the Swiss Financial Market Supervisory Authority. Effective as of $1^{\text {st }}$ January 2015, a fee on early redemptions of time deposits of at least 2 percent is levied.

Our finding of an optimal FTT of 1.6 percent for the United States or 1.5 percent for Germany is likely to be an upper bound for the optimal FTT, since, by construction, in our paper there are no negative effects of a FTT on the primary market and, for example, investment decisions. Furthermore, our model is a closed economy, where agents cannot avoid the FTT by moving to asset markets that have no or a lower FTT. In practice, financial investors have many choices at home and abroad and this will constrain the introduction of FTTs. An open economy, however, does not imply that a FTT will drive away all asset trading. For example, in Switzerland, the tax authority charges a stamp tax (a FTT) equal to 0.15 percent for each transaction in domestically issued CHF bonds and a stamp tax equal to 0.3 percent for each transaction in foreign issued CHF bonds. Even though these rates are considerably smaller than the optimal rates we find for the United States and Germany, the tax income generated by the Swiss stamp duty is large. For Switzerland in 2010, it generated 4.5 percent of the entire federal tax income. ${ }^{4}$

To place our main finding into the relevant context, the basic setup here is a variant of a class of models that is labeled the "new monetarist economics." ${ }^{5}$ This body of literature originated with the seminal paper by Kiyotaki and Wright (1989). Our version is based on Lagos and Wright (2005) and Berentsen et al. (2007). In these new generation models, the Friedman rule is the optimal monetary policy. The Friedman rule maximizes the return on the liquid asset (money) and addresses the problem of an inefficiently low value of money more directly than a FTT. Our paper, therefore, solves a second-best problem in which a FTT can improve welfare away from the Friedman rule.

Implementing the Friedman rule, however, requires taxation, since tax income is needed to subsidize the liquid asset. The FTT, in contrast, generates tax income and, hence, is not subject to this problem. In many monetary models, lump-sum taxation is available and so the necessary funds to implement the Friedman rule can be levied with a nondistortionary tax instrument. If such an instrument is available, enhancing the return of money as proposed by the Friedman rule is clearly a better policy than a FTT. In practice, however, nondistortionary taxation may not be available and a government must resort to distortionary taxation to subsidize the rate of return on the liquid asset. In this case, a well-designed FTT can be a better policy.

\footnotetext{
${ }^{4}$ The stamp duty is levied on many financial products including insurance contracts, stocks, bonds and other financial instruments.

${ }^{5}$ For a discussion of this literature, see Williamson and Wright (2010a and 2010b).
} 
It is well-known that a pecuniary externality is a pricing externality. In an incomplete markets setting, the equilibrium might not be constrained efficient and government intervention can be welfare-improving. In our incomplete market model, there are two pecuniary externalities. First, when agents acquire money they incur disutility today, but spending and hence consumption utility occurs in the future. Since agents discount future utilities, they typically underinvest in money, which results in a value of money that is too low, or, equivalently, a price level that is too high. This is a well-known pecuniary externality that is present in the most basic version of the Lagos and Wright (2005) framework. We add to this basic setting the opportunity to trade liquid for illiquid assets after observing the idiosyncratic liquidity shocks. This opportunity reduces the value of money even more since, as explained above, it reduces the demand for money and hence its value. This second pecuniary externality can be corrected by a FTT.

\section{Literature}

Our paper studies a similar environment as Berentsen et al. (2014). The key finding in this paper is that restricting access to financial markets can be welfare-improving. Here, we find that a FTT can improve the allocation. In both papers, the reason is the presence of a pecuniary externality that arises when agents choose their portfolio of liquid and illiquid assets. There are some important differences, however. First, Berentsen et al. (2014) show that adding trading frictions into an otherwise frictionless competitive market can be welfare-enhancing. In the current paper, we show that trading frictions are necessary for the welfare benefits of a FTT. In particular, we find that in the absence of trading frictions, a FTT is not welfare-improving. Second, the mechanism at work is different. In Berentsen et al. (2014), restricting access to a competitive financial market increases consumption variability across agents. In contrast, in this paper a FTT reduces consumption variability. Third, it might not be feasible to restrict access to financial markets, while imposing a FTT is straightforward. In particular, in Berentsen et al. (2014) access to the financial market is determined randomly, and it is not clear how this can be implemented with financial regulations.

In our model, trading frictions are necessary for the welfare-enhancing effects of a FTT. To capture these frictions, we assume that our financial market is an OTC market, as introduced into the theoretical finance literature by Duffie et al. (2005). ${ }^{6}$ Geromichalos and Herrenbrueck (2016) also develop a model where agents can trade assets of different liquidity in an OTC market. They find that trading in a frictional asset market, as opposed to trading in a competitive asset market, can be welfare-improving. The policy implication is that removing frictions, say by moving from a frictional asset market (OTC-market) to a centrally organized exchange, as often discussed in the aftermath of the financial crisis, can be welfare-decreasing.

\footnotetext{
${ }^{6}$ Recent contributions on OTC markets in this literature include papers by Lagos and Rocheteau (2009), Lagos et al. (2011), and Rocheteau and Wright (2013). Lagos and Rocheteau (2009) extend Duffie et al. (2005) by imposing no restrictions on asset holdings. This allows them to capture the heterogeneous response of agents to changes in market conditions. Lagos et al. (2011) study efficiency of liquidity provisions and government intervention in OTC markets during crises. Rocheteau and Wright (2013) study endogenous agents participation and nonstationary equilibria in OTC markets.
} 
Our framework is part of the rapidly expanding literature labeled the "new monetarist economics." The first paper in this literature that incorporated idiosyncratic liquidity shocks and trading in financial markets is Berentsen et al. (2007). Many more recent papers also add liquidity shocks and financial trading. ${ }^{7}$ However, none of these papers studies the FTT and its implications on welfare. It is also related to the macroeconomic literature on overborrowing. ${ }^{8}$ In this literature, agents do not take into account how their borrowing decisions affect collateral prices (a pecuniary externality), and through them the borrowing constraints of other agents. As a consequence, the equilibrium is characterized by overborrowing, which leads to credit booms and busts. Our paper differs from this literature, because it is not a model of crisis. The pecuniary externality is present in the unique steady-state equilibrium. Furthermore, the pecuniary externality emerges from the portfolio choices and not from borrowing decisions.

There are also many attempts to assess the effects of FTTs empirically. The main issue in this literature is whether a FTT increases or reduces volatility in financial markets. The results from this literature are ambiguous. ${ }^{9}$

To our knowledge, only three papers have so far investigated the implications of a FTT on welfare: $^{10}$ Subrahmanyam (1998), Dow and Rahi (2000) and Dávila (2015). Subrahmanyam (1998) develops a two-period rational expectations model with noisy observations using the Kyle (1985) framework. Subrahmanyam shows that a transaction tax on financial transactions reduces an agent's incentive to acquire information before others do so, and eventually increases welfare. The main argument proposed by Subrahmanyam is that agents spend too much effort on information acquisition. A policy that induces agents to reduce trading, such as a transaction tax, can be socially beneficial. Dow and Rahi (2000) study the welfare effects of a transaction tax in a model with informed and uninformed agents. They show that a tax on transactions made by informed agents can be beneficial both for them and for the uninformed agents. They also show that these results apply when the tax is levied on all transactions, instead of on transactions made by uninformed agents only. Dávila (2015) studies a FTT in a model with belief disagreement. He shows that, when heterogeneous beliefs induce investors to trade too much, it is always optimal to levy a FTT.

\footnotetext{
${ }^{7}$ There is a rapidly growing literature that studies liquidity shocks and financial intermediation in the LagosWright (2005) framework. A sample of these papers are Berentsen and Monnet (2008), Geromichalos and Herrenbrueck (2016), Li (2011), Li and Li (2013), Chiu et al. (2012 and 2016), Chiu and Monnet (2014), and Williamson (2012). Other papers that specifically study OTC markets in this literature are Geromichalos et al. (2016), Lagos and Zhang (2015), and Mattesini and Nosal (2016). For a mechanism design approach to financial intermediation, see $\mathrm{Gu}$ et al. (2013a).

${ }^{8}$ See, for example, Caballero and Krishnamurthy (2003), Lorenzoni (2008), Bianchi and Mendoza (2011), Jeanne and Korinek (2012), Korinek (2012) and Moore (2013). For a more detailed discussion of this literature, see Berentsen et al. (2014).

${ }^{9}$ See Pomeranets (2012) for a detailed discussion of these studies.

${ }^{10}$ There are other theoretical contributions, but they are all concerned with excessive speculation. Our paper is concerned with efficient trade in a monetary economy.
} 


\section{Environment}

Time is discrete and, in each period, there are three markets that open and close sequentially. The first market is an over-the-counter (OTC) secondary bond market, where agents are matched pairwise and trade money for nominal bonds. The second market is a competitive goods market, where agents produce or consume market-2 goods. The third market is a frictionless market, where all agents consume and produce market-3 goods, all financial contracts are redeemed, and new bonds are issued. We label these markets as secondary bond market, goods market, and primary bond market, respectively. All goods are perfectly divisible, and nonstorable in the sense that they cannot be carried from one market to the other.

The economy is populated by a $[0,1]$-continuum of infinitely lived agents. At the beginning of each period, each agent receives an idiosyncratic i.i.d. preference/technology shock that determines whether she is a producer or she is a consumer in the goods market. With probability $n \in(0,1)$, she can produce but not consume, and with probability $1-n$, she can consume but not produce. This shock is introduced in order to obtain a liquidity mismatch and hence a role for asset trading in the secondary bond market. ${ }^{11}$

In the goods market, trading is competitive: Agents take the price of market-2 goods as given and the price clears the market. ${ }^{12}$ A consumer enjoys utility $u(q)$ from $q$ consumption, where $u(q)$ has the standard properties; i.e., $u^{\prime}(q)>0>u^{\prime \prime}(q), u^{\prime}(0)=\infty$, and $u^{\prime}(\infty)=0$. Producers incur a utility $\operatorname{cost} c(q)$ from $q$ production in the goods market. For ease of exposition, we assume linearity of the cost function; i.e., $c(q)=q$.

In the primary bond market, all agents can produce and consume using a linear production technology. In particular, agents can use $h$ units of time to produce $h$ units of market-3 goods. The utility of consuming $x$ units of goods is $U(x)$ where $U^{\prime}(x)>0>U^{\prime \prime}(x), U^{\prime}(0)=\infty$, and $U^{\prime}(\infty)=0$. Agents discount between, but not within, periods. The discount factor between two consecutive periods is $\beta=1 /(1+r)$, where $r>0$ represents the real interest rate. It is routine to show that the first-best quantities satisfy $U^{\prime}\left(x^{*}\right)=1$ and $u^{\prime}\left(q^{*}\right)=1$.

There are two perfectly divisible, storable objects: money and nominal bonds. Both money and bonds are intrinsically useless, and are issued by the central bank in the primary bond market. Bonds are issued at discount, and one unit of bonds pays one unit of money in the nextperiod primary bond market. The central bank has a record-keeping technology over financial transactions. Bonds are intangible objects, and the central bank operates the primary and secondary bond markets and keeps track of ownership. This also allows for the imposition of a FTT.

Trading in the goods market requires a medium of exchange. The frictions that make the use of a medium of exchange in the goods market necessary are specialization in production and

\footnotetext{
${ }^{11}$ This liquidity shock was introduced in Berentsen et al. (2007). In their model, financial intermediation emerges endogenously to mitigate the liquidity mismatch generated by these shocks.

${ }^{12}$ In an earlier version of the paper, agents were subject to search and bargaining frictions in this market as well. Assuming competitive pricing has three implications: First, it eliminates the mark-up inefficiency that arises when agents bargain over the terms of trade. Second, it simplifies the theoretical part of the paper considerably. Third, it does not affect the qualitative result in an important way. For a more exhaustive analysis of different trading protocols in the goods market, see Rocheteau and Wright (2005).
} 
consumption, limited commitment, and a lack of record-keeping. ${ }^{13}$ In our model, only money can serve as a medium of exchange. The reason is that bonds are intangible objects, and so they are incapable of being used as a medium of exchange in the goods market; hence, they are illiquid. Therefore, money is the only means of payment in the goods market. ${ }^{14}$

In the secondary bond market, agents meet according to a matching function $\mathcal{M}[\xi n, \xi(1-n)]$, where the parameter $\xi$ is a scaling variable, which determines the efficiency of the matching process. We assume that $\mathcal{M}$ has constant returns to scale, and is continuous and increasing with respect to each of its arguments. The probability of a meeting for a consumer and a producer are then $\delta \equiv \xi \mathcal{M}(n, 1-n)(1-n)^{-1}$ and $\delta^{p} \equiv \delta(1-n) n^{-1}$, respectively. Once in a meeting, the consumer and producer bargain over the quantity of money and bonds to be exchanged. Specifically, terms of trade in the secondary bond market are determined according to Kalai bargaining. We refer to agents who are matched in this market as active, and to those who are not as passive.

Let $M_{t}$ be the per-capita stock of money and $B_{t}$ the per-capita stock of newly issued bonds at the end of period $t$. Let $\rho_{t}$ denote the price of bonds in the primary bond market. Then, the law of motion of money in period $t$ is given by

$$
M_{t}-M_{t-1}=T+B_{t-1}-\rho_{t} B_{t}-T_{b}
$$

The change in the stock of money at time $t, M_{t}-M_{t-1}$, is affected by four components: the lumpsum money injection $(T>0)$ or withdrawal $(T<0)$, the money created to redeem previously issued bonds, $B_{t-1}$, the money withdrawn from selling newly issued bonds, $\rho_{t} B_{t}$, and the revenues from the FTT in the secondary bond market, $T_{b} .{ }^{15}$ We assume that there is a strictly positive initial stock of money and bonds; i.e., $M_{0}, B_{0}>0$.

\section{Agent's Decisions}

For notational simplicity, the time subscript $t$ is omitted from now on. Next-period variables are indexed by +1 , and previous-period variables are indexed by -1 . In what follows, we study the agents' decisions beginning in the last market (the primary bond market) and then move backwards within a period to the goods market, and finally to the secondary bond market.

\footnotetext{
${ }^{13}$ The essential role of a medium of exchange has been studied, for example, by Kocherlakota (1998) and Wallace (2001). Sanches and Williamson (2010) show that an economy with no memory and monetary exchanges may achieve the same equilibrium allocation as an economy with perfect memory and private credit. Limited commitment is important for this result. In a similar fashion, Gu et al. (2013a and 2013b) study issues related to banking and credit.

${ }^{14}$ An alternative arrangement that would render bonds illiquid is if they can be counterfeited at no cost (Li et al. 2012).

${ }^{15}$ The total amount of tax revenues from FTTs are $T_{b}=\tau \int d_{m}(i) d i$ where $d_{m}(i)$ denotes the amount of money exchanged in the $i$-th meeting in the secondary bond market and $\tau$ is the FTT. We will see that, in a symmetric equilibrium, $d_{m}(i)=d_{m}$, and since there are $(1-n) \delta$ meetings in the secondary bond market, and trade always occurs in a meeting, then $T_{b}=\tau d_{m}(1-n) \delta$.
} 


\subsection{Primary bond market}

In the primary bond market, previous-period bonds are redeemed and agents choose a portfolio of money and newly issued bonds by producing and consuming market-3 goods. An agent entering the primary bond market with $m$ units of money and $b$ units of bonds has the indirect utility function $V_{3}(m, b)$. His decision problem is

$$
V_{3}(m, b)=\underset{x, h, m_{+1}, b_{+1}}{\arg \max }\left[U(x)-h+\beta V_{1}\left(m_{+1}, b_{+1}\right)\right],
$$

subject to

$$
x+\phi m_{+1}+\phi \rho b_{+1}=h+\phi m+\phi b+\phi T .
$$

The first-order conditions with respect to $m_{+1}, b_{+1}$ and $x$ are

$$
\frac{\beta \partial V_{1}}{\partial m_{+1}}=\rho^{-1} \frac{\beta \partial V_{1}}{\partial b_{+1}}=\phi,
$$

and $U^{\prime}(x)=1$, respectively. The term $\frac{\beta \partial V_{1}}{\partial m_{+1}}\left(\frac{\beta \partial V_{1}}{\partial b_{+1}}\right)$ is the marginal benefit of taking one additional unit of money (bonds) into the next period, while $\phi(\rho \phi)$ is the marginal cost. Due to the quasi-linearity of preferences, the choices of $b_{+1}$ and $m_{+1}$ are independent of $b$ and $m$. It is straightforward to show that all agents exit the primary bond market with the same portfolio of bonds and money. The envelope conditions in the primary bond market are

$$
\frac{\partial V_{3}}{\partial m}=\frac{\partial V_{3}}{\partial b}=\phi
$$

According to (5), the marginal value of money and bonds at the beginning of the primary bond market is equal to the price of money in terms of market-3 goods. Note that (5) implies that the value function $V_{3}$ is linear in $m$ and $b$.

\subsection{Goods market}

In the goods market, consumers consume and producers produce the market-2 good. Terms of trade are determined by competitive pricing in this market. Denote $p$ the competitive price for market-2 goods.

Let $V_{2}^{p}(m, b)$ be the value function of a producer entering the goods market with $m$ units of money and $b$ units of bonds. His problem is to choose the amount of production, $q_{p}$, such that his lifetime utility is maximized; i.e.,

$$
V_{2}^{p}(m, b)=\max _{q_{p}}-q_{p}+V_{3}\left(m+p q_{p}, b\right) .
$$

The first-order condition for a producer in the goods market is $1 / p=\partial V_{3} / \partial m$. Using (5), the first-order condition can be rewritten as

$$
1=p \phi
$$


Envelope conditions are

$$
\frac{\partial V_{2}^{p}}{\partial m}=\frac{\partial V_{2}^{p}}{\partial b}=\phi
$$

where, again, we have used (5).

Let $V_{2}^{c}(m, b)$ be the value function of a consumer entering the goods market with $m$ units of money and $b$ units of bonds. Then, his problem in the goods market is

$$
V_{2}^{c}(m, b)=\max _{q_{c}} u\left(q_{c}\right)+V_{3}\left(m-p q_{c}, b\right),
$$

subject to $m-p q_{c} \geq 0$. Let $\lambda$ be the Lagrange multiplier for this constraint. A consumer in the goods market decides how much to consume, $q_{c}$, taking the price of the market-2 good as given, and subject to the constraint that he cannot spend more money than he has. Using (5) and (6), the first-order condition satisfies

$$
\phi u^{\prime}\left(q_{c}\right)=\phi+\lambda .
$$

From (8), consumption is efficient, $u^{\prime}\left(q_{c}\right)=1$, if the consumer does not spend all his money in the goods market; i.e., $\lambda=0$. In contrast, consumption is inefficient, $u^{\prime}\left(q_{c}\right)>1$, if the consumer's cash constraint binds. The envelope conditions for a consumer in the goods market are

$$
\frac{\partial V_{2}^{c}}{\partial m}=\phi u^{\prime}\left(q_{c}\right) \quad \text { and } \quad \frac{\partial V_{2}^{c}}{\partial b}=\phi .
$$

All the expressions above hold for any agent entering the goods market with a portfolio $(m, b)$ of assets. In the next subsection, we show that active agents and passive agents enter the goods market with a different portfolio. This generates consumption heterogeneity in this market.

\subsection{Secondary bond market}

In the secondary bond market, consumers and producers are matched pairwise, and the gains from trade are split according to the proportional bargaining solution, introduced by Kalai (1977). To derive the terms of trade, one can consider the case where the consumer chooses the terms of trade in order to maximize his payoff subject to the constraint that the producer receives a given fraction of the total surplus (see Aruoba et al., 2007). In particular, he chooses the quantities $\left(d_{m}, d_{b}\right)$, where $d_{m}$ is the quantity of money he receives for $d_{b}$ units of bonds.

Transactions in the secondary bond market are subject to a proportional tax $\tau$. If the producer accepts the offer, $d_{b}$ units of bonds and $d_{m}$ units of money change hands, and the consumer pays $\tau d_{m}$ units of money to the government. We assume that the government operates the secondary bond market and as such can perfectly enforce tax payment. Participation in the secondary bond market is voluntary so that agents always have the option to avoid the tax by not trading. This contrasts with the scenario for a lump-sum tax where the assumption of perfect enforcement means that agents always have to pay it. ${ }^{16}$

\footnotetext{
${ }^{16}$ Andolfatto (2013) studies the case where lump-sum taxation must satisfy participation constraints, which limits the government's ability to run the Friedman rule.
} 
The consumer's problem in a match in the secondary bond market is

$$
\max _{d_{m}, d_{b}} \Delta^{c}
$$

subject to

$$
\begin{aligned}
(1-\eta) \Delta^{c} & \leq \eta \Delta^{p} \\
\phi b-\phi d_{b} & \geq 0 \\
\phi m-\phi d_{m} & \geq 0
\end{aligned}
$$

where $\eta \in[0,1]$ is the consumer's bargaining weight in a meeting, and $\Delta^{c}$ and $\Delta^{p}$ are the consumer's and producer's net surplus. The first constraint in the consumer's problem is the Kalai constraint. The second constraint means that a consumer cannot deliver more bonds than he has, and the third constraint means that a producer cannot deliver more money than she has.

In the Appendix, we derive expressions for $\Delta^{c}$ and $\Delta^{p}$. Using these expressions, the consumer's problem in the secondary bond market can be rewritten as follows: ${ }^{17}$

$$
\max _{d_{m}, d_{b}}\left[u\left(\hat{q}_{c}\right)-u\left(q_{c}\right)\right]-\phi d_{b}
$$

subject to

$$
\begin{aligned}
\phi d_{b} & \geq(1-\eta)\left[u\left(\hat{q}_{c}\right)-u\left(q_{c}\right)\right]+\eta \phi d_{m} \\
\phi b & \geq \phi d_{b} \\
\phi m & \geq \phi d_{m}
\end{aligned}
$$

where $\hat{q}_{c}$ and $q_{c}$ are the consumption quantities of an active consumer and passive consumer, respectively. The first constraint is again the Kalai constraint. In any equilibrium, it has to hold with equality, and it is therefore convenient to solve it for $d_{b}$ to obtain

$$
\phi d_{b}=(1-\eta)\left[u\left(\hat{q}_{c}\right)-u\left(q_{c}\right)\right]+\eta \phi d_{m} .
$$

Use (10) to eliminate $\phi d_{b}$ from the objective function and the second inequality, and rewrite the consumer's problem as follows:

$$
\max _{d_{m}} \eta\left\{\left[u\left(\hat{q}_{c}\right)-u\left(q_{c}\right)\right]-\phi d_{m}\right\}
$$

\footnotetext{
${ }^{17}$ The solution to this problem always satisfies the producer's participation constraint, $\Delta^{p} \geq 0$. In contrast, the consumer's participation constraint, $\Delta^{c} \geq 0$, may not be satisfied. This is, in particular, the case if the tax is high and/or inflation low, which reduces the benefits from having the secondary bond market. In this case, there is no trading and the market shuts down. In what follows, we assume that the tax (or the inflation) is such that there is trading, and later on we verify under which conditions $\Delta^{c} \geq 0$.
} 
subject to

$$
\begin{aligned}
\phi b-(1-\eta)\left[u\left(\hat{q}_{c}\right)-u\left(q_{c}\right)\right]-\eta \phi d_{m} & \geq 0 \\
\phi m-\phi d_{m} & \geq 0 .
\end{aligned}
$$

Note that the expression in the curly bracket in the objective function is the total surplus of the match $\Delta^{p}+\Delta^{c}$. Thus, the Kalai proportional solution maximizes the total surplus and is hence efficient.

Denote $\lambda^{c}$ and $\lambda^{p}$ the Lagrange multipliers for constraints (12) and (13), respectively. As we will demonstrate, the nature of the equilibrium will depend on whether these constraints are binding or not. The first-order condition in the secondary bond market is

$$
\eta\left[u^{\prime}\left(\hat{q}_{c}\right) \frac{\partial \hat{q}_{c}}{\partial d_{m}}-\phi\right]-\lambda^{c}\left[(1-\eta) u^{\prime}\left(\hat{q}_{c}\right) \frac{\partial \hat{q}_{c}}{\partial d_{m}}+\eta \phi\right]-\phi \lambda^{p}=0 .
$$

Finally, the value function in the primary bond market satisfies

$$
V_{1}(m, b)=n V_{1}^{p}(m, b)+(1-n) V_{1}^{c}(m, b),
$$

where

$$
\begin{aligned}
V_{1}^{c}(m, b) & =\delta \hat{V}_{2}^{c}\left(m+d_{m}(1-\tau), b-d_{b}\right)+(1-\delta) V_{2}^{c}(m, b) \\
V_{1}^{p}(m, b) & =\delta^{p} \hat{V}_{2}^{p}\left(m-d_{m}, b+d_{b}\right)+\left(1-\delta^{p}\right) V_{2}^{p}(m, b) .
\end{aligned}
$$

The value function of a consumer at the beginning of the secondary goods market, (16), is given by the value function of an active consumer times the probability of a consumer being active in this market, $\delta \hat{V}_{2}^{c}$, plus the value function of a passive consumer times the probability of a consumer being passive, $(1-\delta) V_{2}^{c}$. The value function (17) refers to a producer and has a similar interpretation.

\section{$5 \quad$ Monetary Equilibrium}

We focus on symmetric, stationary monetary equilibria, where all agents follow identical strategies and where real variables are constant over time. Let $\zeta \equiv B / B_{-1}$ denote the gross growth rate of bonds, and let $\gamma \equiv M / M_{-1}$ denote the gross growth rate of the money supply. In a stationary monetary equilibrium, the real stock of money must be constant; i.e., $\phi M=\phi_{+1} M_{+1}$, implying that $\gamma=\phi / \phi_{+1}$. Furthermore, the real amount of bonds must be constant; i.e., $\phi B=\phi_{-1} B_{-1}$, implying that $\zeta=\gamma$.

Market clearing in the goods market implies that aggregate consumption is equal to aggregate production; i.e., $(1-n)\left[\delta \hat{q}_{c}+(1-\delta) q_{c}\right]=n q_{p}$. Aggregate consumption is given by the consumption of active consumers times their measure, $\hat{q}_{c}(1-n) \delta$, plus the consumption of passive consumers times their measure, $q_{c}(1-n)(1-\delta)$. Aggregate production is given by the production of producers times their measure, $q_{p} n$. Again, note that production in the goods 
market does not depend on a producer's portfolio, and so all producers produce the same quantity of goods, $q_{p}$. To make the notation simpler, we omit the subscript " $c$ " in the consumed quantities and relabel $\hat{q}_{c}$ and $q_{c}$ as $\hat{q}$ and $q$, respectively. Hence, the market clearing condition satisfies

$$
(1-n)[\delta \hat{q}+(1-\delta) q]=n q_{p}
$$

In what follows, we focus on two cases. In the first case, labeled type-I equilibrium, the constraints (12) and (13) do not bind (i.e., $\lambda^{c}=\lambda^{p}=0$ ). In the second case, labeled type-II equilibrium, the producer's cash constraint binds and the consumer's bond constraint does not bind (i.e., $\lambda^{p}>0$ and $\lambda^{c}=0$ ). Further below, we calibrate the model to U.S. data and find that these are the relevant cases. ${ }^{18}$

All equilibria involve the derivation of the marginal values of money and bonds from equation (15). Furthermore, the Kalai equation (10) and the first-order condition in the secondary bond market (14) play a key role. This last equation can be written as follows:

$$
\eta\left[(1-\tau) u^{\prime}(\hat{q})-1\right]=\lambda^{p}+\lambda^{c}\left[(1-\eta)(1-\tau) u^{\prime}(\hat{q})+\eta\right],
$$

where we have used the budget constraint in the goods market; i.e., $m+d_{m}(1-\tau)=p \hat{q}$, to replace $\partial \hat{q} / \partial d_{m}=\phi(1-\tau)$.

\subsection{Type-I equilibrium}

In a type-I equilibrium, an active consumer's bond constraint does not bind, and an active producer's cash constraint does not bind. A type-I equilibrium can be characterized by the three equations stated in Proposition 1. All proofs are in the Appendix.

Proposition 1 A type-I equilibrium is a time-invariant path $\{\hat{q}, q, \rho\}$ satisfying

$$
\begin{aligned}
1 & =u^{\prime}(\hat{q})(1-\tau) \\
\frac{\gamma}{\beta} & =(1-n)\left[\delta\left\{u^{\prime}(q)+\eta\left[u^{\prime}(\hat{q})-u^{\prime}(q)\right]\right\}+(1-\delta) u^{\prime}(q)\right]+n, \\
\frac{\rho \gamma}{\beta} & =1 .
\end{aligned}
$$

Equation (20) is derived from the first-order condition (19). The meaning of this equation is that the cost of acquiring one additional unit of money in a meeting in the secondary bond market has to be equal to its benefit.

Equation (21) is derived from the marginal value of money in the secondary bond market. The right-hand side of (21) is the marginal benefit of money at the beginning of the period. With probability $(1-n) \delta$, the agent is an active consumer and the marginal benefit of money is $u^{\prime}(q)+\eta\left[u^{\prime}(\hat{q})-u^{\prime}(q)\right]$. Note that the term $\left[u^{\prime}(\hat{q})-u^{\prime}(q)\right]$ is negative, since an active consumer holds more money in the goods market. Note further that a consumer with a higher bargaining

\footnotetext{
${ }^{18}$ The other possible values of the multipliers (i.e., $\lambda^{c}>0$ and $\lambda^{p}=0$ ) are analyzed in a Supplementary Appendix that is available on request.
} 
weight, $\eta$, holds more money, and so the marginal benefit of money is decreasing in $\eta$. With probability $(1-n)(1-\delta)$, the agent is an passive consumer, since he has no match and his marginal utility is $u^{\prime}(q)$. With probability $n$ the agent is a producer and her marginal utility is 1 . The left-hand side of (21) represents the marginal cost of acquiring one additional unit of money in the primary bond market. Equation (22) is the Fisher equation. It reflects the fact that the benefit of taking one additional unit of bonds into the secondary bond market must be equal to the marginal cost of acquiring it in the primary bond market.

\subsection{Type-II equilibrium}

In a type-II equilibrium, an active consumer's bond constraint does not bind, and an active producer's cash constraint binds. The following Proposition 2 characterizes the type-II equilibrium.

Proposition 2 A type-II equilibrium is a time-invariant path $\{\hat{q}, q, \rho\}$ satisfying

$$
\begin{aligned}
\hat{q}= & (2-\tau) q, \\
\frac{\gamma}{\beta}= & (1-n)\left\{\delta\left\{u^{\prime}(q)+\eta\left[u^{\prime}(\hat{q})-u^{\prime}(q)\right]\right\}+(1-\delta) u^{\prime}(q)\right\} \\
& +n\left\{\delta^{p}\left[(1-\eta)(1-\tau) u^{\prime}(\hat{q})+\eta\right]+\left(1-\delta^{p}\right)\right\} \\
\frac{\gamma \rho}{\beta}= & 1 .
\end{aligned}
$$

To derive equation (23), we compare the budget constraint of an active consumer with the budget constraint of a passive consumer. Furthermore, we use the fact that a producer transfers all her money to the active consumer. The interpretations of (24) and (25) are similar to their counterparts in Proposition 1. It is interesting to compare (24) to its counterpart (21). They are equal except for the marginal value of money for the producer. In (21), the producer's marginal value of money is 1 , while in $(24)$ it is $\delta^{p}\left[(1-\eta)(1-\tau) u^{\prime}(\hat{q})+\eta\right]+\left(1-\delta^{p}\right)>1$. The reason is that, in the type-II equilibrium, the producer's cash constraint is binding $\left(\lambda^{p}>0\right)$, and so the rate of return on money holdings is strictly positive.

\subsection{Regions of Existence}

Proposition 3 characterizes two non-overlapping regions in which the two types of equilibria exist. Let $\gamma_{1}$ denote the value of $\gamma$ such that $\hat{q}=q$ holds in the type-I equilibrium. Furthermore, let $\gamma_{2}$ denote the value of $\gamma$ such that equations (21) and (24) hold simultaneously. In the proof of Proposition 3, we show that such values exist and that they are unique. Furthermore, we show under which conditions $\beta \leq \gamma_{1} \leq \gamma_{2}<\infty$.

Proposition 3 If $\gamma_{1} \leq \gamma<\gamma_{2}$, equilibrium prices and quantities are characterized by Proposition 1; and if $\gamma_{2} \leq \gamma$, they are characterized by Proposition 2.

In the type-I equilibrium $\left(\gamma_{1} \leq \gamma<\gamma_{2}\right)$, consumers and producers are unconstrained in the secondary bond market (i.e., $\left.\lambda^{c}=\lambda^{p}=0\right)$. In the type-II equilibrium $\left(\gamma_{2} \leq \gamma\right)$, active consumers 
are unconstrained, but the constraint on money holdings of active producers binds (i.e., $\lambda^{c}=0$, $\left.\lambda^{p}>0\right)$. Thus, in both types of equilibria active consumers do not sell all their bond holdings and thus the price of bonds in the primary bond market, $\rho$, must equal the fundamental value of bonds, $\beta / \gamma$.
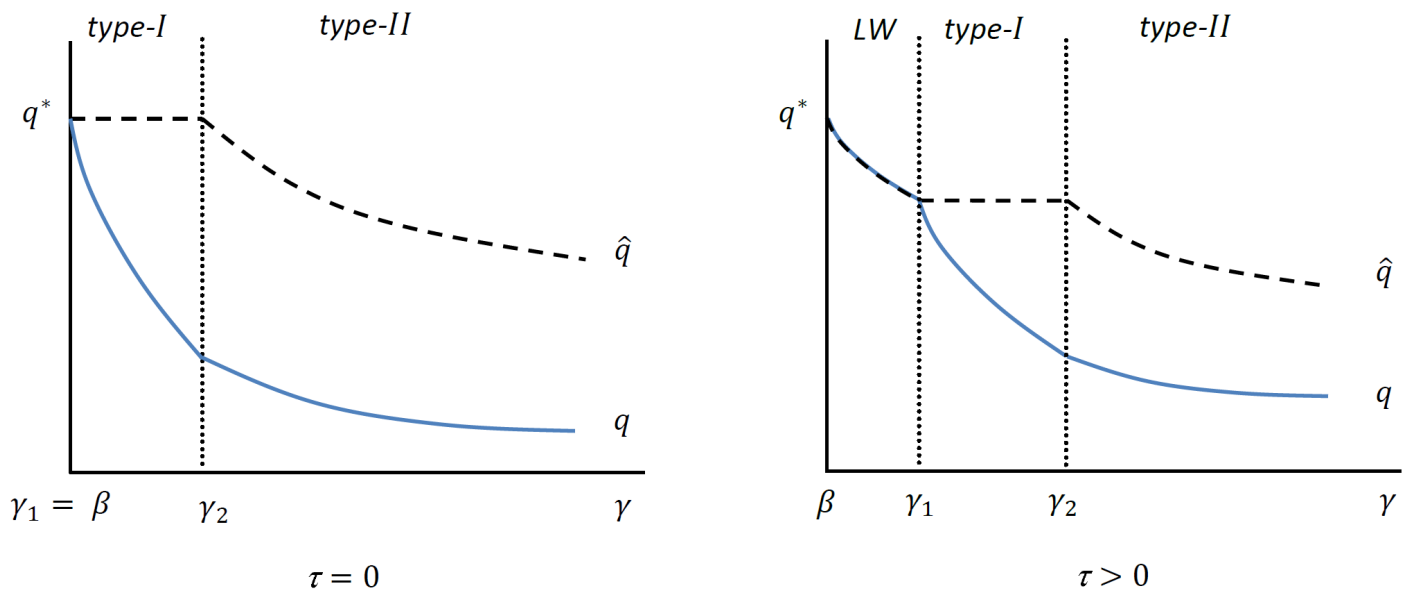

Figure 1: Consumed Quantities for $\tau=0$ And $\tau>0$.

Figure 1 shows the consumed quantities as a function of $\gamma^{19}$ For $\tau=0$ and $\gamma_{1}=\beta \leq \gamma<\gamma_{2}$, the economy is in the type-I equilibrium, where active consumers obtain the first-best quantity; i.e., $\hat{q}=q^{*}$, while passive consumers obtain $q \leq q^{*}$. For $\tau>0$ and $\beta<\gamma_{1} \leq \gamma<\gamma_{2}$, both consumption quantities are less than $q^{*}$ (see the graph on the right-hand side of Figure 1). For $\beta \leq \gamma<\gamma_{1}$, active agents are better off by not trading in the secondary bond market; i.e., $\Delta^{c}<0$. In this case, the quantities are equal and correspond to the consumption quantities obtained in the standard Lagos and Wright (2005) framework (see the region labeled LW in the graph on the right-hand side of Figure 1). ${ }^{20}$ For increasing values of $\tau$, the critical values $\gamma_{1}$ and $\gamma_{2}$ both move to the right. Finally, for $\gamma_{2}<\gamma$ both quantities are smaller than $q^{*}$ and decreasing in $\gamma$.

\section{Optimal Tax}

The main result of our paper is that imposing a FTT in the secondary bond market can be welfare-increasing. In this section, we show under which conditions this is the case, and we

\footnotetext{
${ }^{19}$ Throughout the paper when we consider a change in the FTT, we assume that the additional tax income is redistributed lump-sum to the agents in the primary bond market. This means from (1), that a change in the FTT has no effect on the inflation rate.

${ }^{20}$ In this region, the consumption quantity satisfies $\frac{\gamma}{\beta}=(1-n) u^{\prime}(q)+n$ and $\hat{q}=q$. The bond price is at its fundamental value $\frac{\gamma \rho}{\beta}=1$.
} 
provide intuition for the result. Let $\mathcal{W}$ be the expected life-time utility of the representative agent at the end of the period. Then, welfare $\mathcal{W}$ can be written as follows:

$$
(1-\beta) \mathcal{W}=(1-n)\{\delta[u(\hat{q})-\hat{q}]+(1-\delta)[u(q)-q]\}+U\left(x^{*}\right)-x^{*}
$$

where the term in the curly brackets is an agent's expected period utility in the goods market, and $U\left(x^{*}\right)-x^{*}$ is the agent's period utility in the primary bond market. Differentiating (26) with respect to $\tau$ yields

$$
\frac{1-\beta}{1-n} \frac{d \mathcal{W}}{d \tau}=\delta\left[u^{\prime}(\hat{q})-1\right] \frac{d \hat{q}}{d \tau}+(1-\delta)\left[u^{\prime}(q)-1\right] \frac{d q}{d \tau}
$$

The welfare effect depends on the derivatives $\frac{d \hat{q}}{d \tau}$ and $\frac{d q}{d \tau}$. In the type-I equilibrium, from (20), we have $\frac{d \hat{q}}{d \tau}=\frac{1}{(1-\tau)^{2} u^{\prime \prime}(\hat{q})}<0$, and, from $(21)$, we have $\frac{d q}{d \tau}=-\frac{\delta \eta}{(1-\delta \eta)(1-\tau)^{2} u^{\prime \prime}(q)}>0$. Thus, whether a FTT is welfare-improving depends on which of the two effects dominates.

Proposition 4 In the type-I equilibrium, if

$$
\Theta(q, \hat{q})=\left[\frac{(\eta-\eta \delta) u^{\prime \prime}(\hat{q})}{(1-\eta \delta) u^{\prime \prime}(q)}\right]\left[\frac{u^{\prime}(q)-1}{u^{\prime}(\hat{q})-1}\right]>1,
$$

then welfare is increasing in $\tau$.

Proposition 4 formulates a condition under which it is welfare-improving to increase the FTT in the type-I equilibrium. In general, the first term is smaller than 1 and the second term is larger than 1 . The second term approaches infinity as $\hat{q} \rightarrow q^{*}$, which means that for some preferences and technology parameters the second term dominates the first term. ${ }^{21}$

The search frictions play a crucial role for this result. From $(28), \Theta(q, \hat{q})$ is decreasing in $\delta$ and approaches 0 as $\delta \rightarrow 1$. In the absence of search frictions $(\delta=1)$, all consumers trade in the secondary bond market, and so all consumers obtain the same consumption $\hat{q}$ in the goods market. In this case, adding a FTT is strictly welfare-decreasing, since it lowers consumption for all consumers.

This last observation also clarifies why a FTT can be welfare-increasing. In the type-I equilibrium, we have $q<\hat{q}$. Increasing $\tau$ increases $q$ and decreases $\hat{q}$. Thus, the tax has a redistributional effect. The question is why does it increase $q$ ? The reason is straightforward. The role of the secondary bond market is to allocate idle money from producers to consumers. In doing so, this market provides insurance to agents against the liquidity shock of becoming a consumer. The drawback of this insurance is that it reduces the incentive to self-insure against the liquidity shocks. This lowers the demand for money in the primary bond market, which depresses its value. This effect can be so strong that it can be optimal to impose a FTT in the secondary bond market.

\footnotetext{
${ }^{21}$ In particular, consider an initial FTT of $\tau=0, \delta<1$ and $\gamma_{1}<\gamma<\gamma_{2}$. In this case, we have $\hat{q}=q^{*}$, and so this condition is satisfied, since $\lim _{\hat{q} \rightarrow q^{*}} \Theta\left(q, q^{*}\right)=\infty$.
} 


\section{Discussion}

In this section, we discuss the role of search frictions for the optimal FTT, the Friedman rule, and other issues of relevance for our analysis.

Role of search frictions. We have shown above that search frictions are needed in order for a FTT to be welfare-increasing. In order to verify that search frictions (instead of bargaining frictions) are necessary for the welfare benefit of a FTT, we have also derived a version of the model where pricing in the secondary bond market is competitive. To mimic search frictions, we assume that traders have random access to the secondary bond market. ${ }^{22}$ For this competitive pricing model and for the type-I equilibrium, we find a similar condition to condition (28). ${ }^{23}$ Namely,

$$
\Theta(q, \hat{q})=\left[\frac{u^{\prime \prime}(\hat{q})}{u^{\prime \prime}(q)}\right]\left[\frac{u^{\prime}(q)-1}{u^{\prime}(\hat{q})-1}\right]>1 .
$$

The second term is always larger than 1 , since consumption with access to this market is larger than that with no access; i.e., $\hat{q}>q$. Since $u^{\prime \prime}(\hat{q})>u^{\prime \prime}(q)$, the above inequality is always satisfied.

The optimal taxation is defined as the value of $\tau$ that maximizes ex-ante welfare, which is given by (26). How does the optimal FTT affect the consumed quantities in the goods market, when we assume that the secondary bond market is competitive? In contrast to Kalai bargaining, the consumed quantities of active and passive agents equal each other for $\tau=\tau^{*}$; i.e., with competitive pricing and random access to the secondary bond market $(\eta=1$ and $\delta<1)$, the FTT eliminates any consumption variability, which is shown in the left-hand chart of Figure 2.

\footnotetext{
${ }^{22}$ For competitive pricing, we assume that $n=0.5$. In this case $\delta=\delta^{p}$ and so consumers and producers have the same access probability.

${ }^{23}$ We have rewritten the model assuming competitive pricing and limited participation. For the type-I equilibrium, we get the same allocation as for the OTC market under Kalai bargaining with $\eta=1$. For the type-II equilibrium, the expressions are not quite the same, but since we focus on the type-I equilibrium in the calibration, we do not report this here. The proof is available on request.
} 

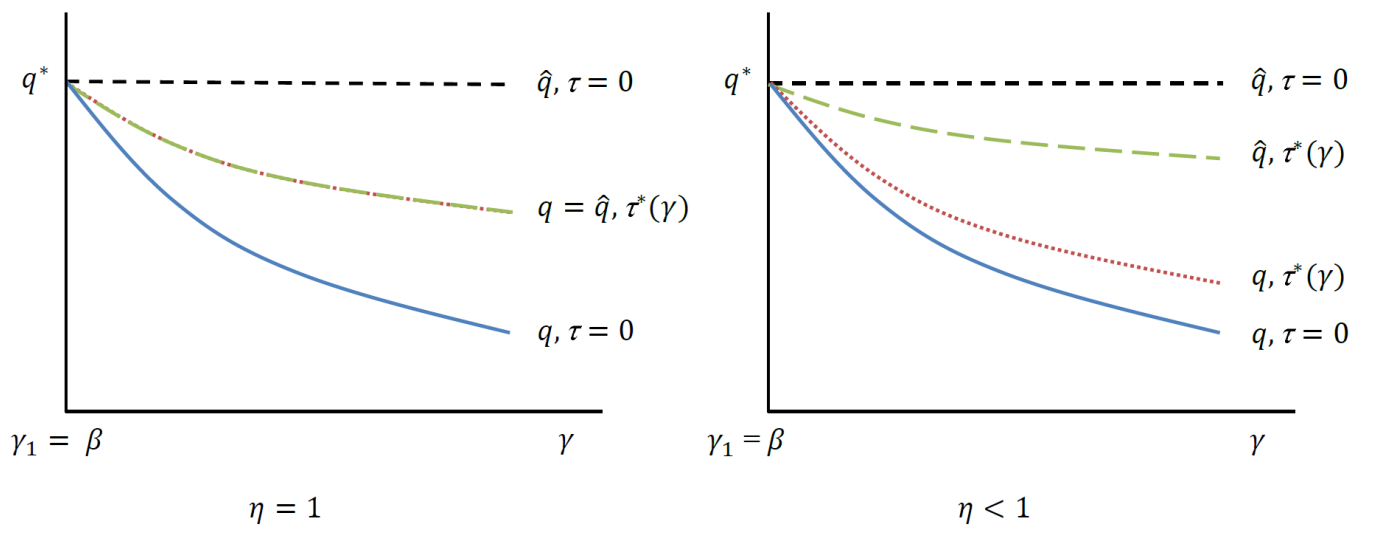

Figure 2: Consumed Quantities for $\eta=1$ And $\eta<1$.

The green and red line represent the consumed quantities of active and passive consumers, respectively, for $\tau=\tau^{*}(\gamma)$, which is calculated for each inflation rate $\gamma$. Note that, for $\tau=\tau^{*}(\gamma)$, we have $\hat{q}=q$ and thus $\gamma_{1}=\beta$. The chart on the right-hand side of the above figure shows the consumed quantities for Kalai bargaining when $\eta<1$. In this case, consumption variability is also reduced under the optimal tax rate (see the right-hand diagram in Figure 2), but we have $\hat{q}>q$.

The Friedman rule and paying interest on money For $\tau=0$ and $\gamma=\beta$, we have $q=\hat{q}=q^{*}$ (see the graph on the left-hand side of Figure 1). That is, the Friedman rule $(\gamma=\beta)$ implements the first-best allocation. In this case, there is obviously no welfare-enhancing role for a FTT. There is not even a role for a secondary bond market, since holding cash is costless and agents do not need to economize their cash holdings.

An alternative policy is to pay interest on money, as proposed in Andolfatto (2010). Both policies enhance the return on holding money and address the problem of an inefficiently low value of money more directly than a FTT. Both policies, however, require some form of taxation, since tax income is needed to subsidize the liquid asset. The FTT, in contrast, generates tax income and, hence, is not subject to this problem. In this paper, we abstract from this well-known result and ask under which conditions, away from the Friedman rule, can it be welfare-enhancing to impose a transaction tax.

Pecuniary externality. As discussed in the introduction, it is well-known that a pecuniary externality is a pricing externality. In a complete market setting, the resulting equilibrium is still Pareto efficient. In contrast, with incomplete markets the resulting equilibrium might not be constrained efficient, and government intervention can be welfare-improving (see Greenwald and 
Stiglitz, 1986). In our incomplete market model, there are two pecuniary externalities. First, when agents acquire money they incur disutility today, but spending and hence consumption utility occurs in the future. Since agents discount future utilities, they typically underinvest in money, which results in a value of money that is too low or, equivalently, a price level that is too high. This is a well-known pecuniary externality that is present in the most basic version of the Lagos and Wright (2005) framework. In that framework and in our model, the equilibria can be Pareto-ranked in the gross growth rate of the money supply $\gamma$, and the Friedman rule $\gamma=\beta$ implements the first-best allocation.

We add to this basic setting the opportunity to trade liquid for illiquid assets after observing the idiosyncratic liquidity shocks in a secondary bond market. Away from the Friedman rule, this opportunity reduces the value of money even more, since, as explained before, it reduces the demand for money and hence its value. This second pecuniary externality can be corrected by a FTT. Another way to look at this second pecuniary externality is that liquidity shares the characteristics of a public good. Under this view, liquidity is a public good, holding liquidity is costly, and market participants attempt to free-ride on the liquidity holdings of other market participants. As a result, there is an underprovision of liquidity.

As mentioned before, the Friedman rule can address both pecuniary externalities as explained above. Therefore, this paper solves a second-best problem in which a transaction tax can improve welfare when the Friedman rule is not in place. ${ }^{24}$

Other assets. Our analysis should apply to any market where an illiquid asset can be traded for a liquid asset. For simplicity, we call the liquid asset money and the illiquid asset is a risk-free, one-period, government bond. However, the model can be extended to alternative assets such as stocks, T-bills, Muni bonds, and corporate bonds. An analysis of corporate bonds would be of interest, since it would introduce a potential problem of a FTT. A FTT can potentially adversely affect the primary market by making it more difficult to finance investment projects by issuing bonds. In such a case, the benefits of a FTT need to be compared to the potentially negative effects on the stock of capital in the economy.

Taxing the producers. We have also studied the case where the FTT is paid by the producer and not by the consumer. Although some expressions are different, our results still hold. For example, confining our analysis to the type-I equilibrium, the equilibrium equations become

$$
\begin{aligned}
1 & =u^{\prime}(\hat{q})-\tau \\
\frac{\gamma}{\beta} & =(1-n)\left\{\delta\left\{u^{\prime}(q)+\eta\left[u^{\prime}(\hat{q})-u^{\prime}(q)\right]\right\}+(1-\delta) u^{\prime}(q)\right\}+n \\
\frac{\rho \gamma}{\beta} & =1
\end{aligned}
$$

\footnotetext{
${ }^{24}$ A transaction tax cannot implement the first-best, unlike monetary policy, because, although it helps with liquidity provision, it also distorts financial trading that should have happened.
} 
where $\tau$ represents the tax on the producer. It is easy to show that Proposition 4 still holds when producers bear all the tax burden. ${ }^{25}$

Societal benefits of illiquid bonds. In our model, money and bonds are risk-free nominal instruments issued by the central bank. They only differ in terms of liquidity: the former is a liquid asset, while the latter is an illiquid asset. Kocherlakota (2003) shows that making bonds illiquid is an optimal arrangement. The reason is straightforward as explained by Kocherlakota (2003, p. 184): "If bonds are as liquid as money, then people will only hold money if nominal interest rates are zero. But then the bonds can just be replaced by money: there is no difference between the two instruments at all." If there is no difference between the two instruments, then the allocation is unaffected by the presence of a second instrument, since a change in the stock of identical nominal assets is neutral in this class of models. Berentsen and Waller (2011) show that the Kocherlakota (2003) result extends to steady states.

Other reasons for financial trading. Finally, in their original paper about OTC markets, Duffie et al. (2005) offer several reasons of why agents may trade in these markets; i.e., for liquidity, portfolio diversification, speculation, and hedging. In our paper, the only reason is a shock to liquidity needs. We believe that adding these additional motives for trading in financial markets will affect our results quantitatively but not qualitatively. Furthermore, OTC markets are not only characterized by bilateral trade and private negotiations, but they are also characterized by intermediation. That is, very often trade does not happen directly between investors, but it happens through dealers or market-makers. We do not model such intermediation but, from our analysis, we believe that if such intermediaries lower, but do not eliminate, the trading frictions, our results are affected quantitatively but not qualitatively.

Market for borrowing Throughout the paper, we assume that liquidity reallocation occurs through an exchange of a government security for money. In doing so, we have completely shut down any possibility of the emergence of a private market for borrowing and saving that reallocates liquidity such as in Berentsen et al. (2007). This missing market raises serious concerns about the societal benefits of a FTT. Could such a market indeed replace our secondary bond market and make a FTT obsolete?

The answer is no. When agents have limited commitment, a private market for borrowing and lending does not work well in a low interest rate environment. The reason is that the borrowing constraint is very tight for low interest rates. This has been demonstrated in Berentsen et al. (2007). Moreover, Berentsen et al. (2016) calibrate a model with limited commitment to several developed countries and find that limited commitment is indeed a serious issue and that it is visible in the aggregate money demand curve.

\footnotetext{
${ }^{25}$ To see this, note that $\frac{d \hat{q}}{d \tau}=\frac{1}{u^{\prime \prime}(\hat{q})}<0$ from (29), and $\frac{d q}{d \tau}=-\frac{\delta \eta}{(1-\delta \eta) u^{\prime \prime}(q)}>0$ from (30). Plugging these terms into (27) yields $\frac{1-\beta}{(1-n)} \frac{d \mathcal{W}}{d \tau}=\frac{\delta\left[u^{\prime}(\hat{q})-1\right]}{u^{\prime \prime}(\hat{q})}\left\{1-\frac{(1-\delta)\left[u^{\prime}(q)-1\right]}{\delta\left[u^{\prime}(\hat{q})-1\right]} \frac{\delta \eta u^{\prime \prime}(\hat{q})}{[\delta(1-\eta)+1-\delta] u^{\prime \prime}(q)}\right\}$. Now, $\frac{\delta\left[u^{\prime}(\hat{q})-1\right]}{u^{\prime \prime}(\hat{q})}<0$. Hence, $\frac{d \mathcal{W}}{d \tau}>0$ iff (28) holds.
} 
In the next section, we will show that the societal benefits of a FTT exactly occur at low interest rates. Therefore, we think that a private market for borrowing and saving would not affect our qualitative results.

\section{Quantitative Analysis}

In the theory section, we have shown that it can be welfare-improving to impose a FTT. Such a tax makes trading in the secondary bond market less attractive, which results in an increase in the demand for money and its price. The optimal tax rate depends on preferences and technology. In the following, we calibrate the model to U.S. data to obtain estimates for the optimal FTT.

We choose a model period as one year. The functions $u(q), c(q)$, and $U(x)$ have the forms $u(q)=A q^{1-\alpha} /(1-\alpha), c(q)=q$, and $U(x)=\log (x)$, where $A$ is a utility parameter and $\alpha$ is the relative risk aversion. It also measures the elasticity of substitution between consumption in the goods market and the primary bond market. The parameters to be identified are as follows: (i) preference parameters: $(\beta, A, \alpha)$; (ii) financial market parameters: $(n, \delta, \eta)$; (iii) policy parameters: $(\gamma, \tau)$.

To identify these parameters, we use U.S. data from the first quarter of 1995 to the fourth quarter of 2013. All data sources are provided in the Appendix. The calibration targets are shown in Table 1.

Table 1: Calibration targets for the United States

\begin{tabular}{ll}
\hline \hline Target Description & Target Value \\
\hline Average real interest rate & 0.021 \\
Average inflation rate & 0.024 \\
Average velocity of money & 6.30 \\
Average elasticity of money demand & $-0.21(0.014)$ \\
\hline
\end{tabular}

The parameters $\gamma$ and $\beta$ can be set equal to their targets. The gross growth rate of the money supply $\gamma$ matches the average change in the consumer price index. The discount factor $\beta$ is set such that the model's real interest rate matches the real interest rate in the data, which is measured as the difference between the long-term government bond yield and the change in the consumer price index. ${ }^{26}$

The financial market characteristics are captured by the meeting probabilities $\delta$ and $\delta^{p}$ and the bargaining weight $\eta$. To identify a reasonable estimate for $\eta$, we need evidence from direct buyer-seller matching platforms. Direct buyer-seller matching platforms are, for instance, UBS Bond Port, Liquidnet Fixed Income, and HSBC Credit Place. In 2015, UBS Bond Port reached a trading volume of more than USD 30 billion with more than 10,000 bonds being tradable.

\footnotetext{
${ }^{26}$ Related studies work with the yield on AAA bonds, which consists of bonds with remaining maturities of as close as possible to 30 years. As we analyse Germany in the robustness section, we decided to use the long-term government bond yield, which is available for the U.S. and for Germany, and consists of government bonds with a remaining maturity of 10 years.
} 
According to findings of UBS, participants tend to trade at the mid-price, which suggests that $\eta$ is close to $0.5 .^{27}$ In the baseline calibration, we therefore set $\eta=0.5$. We also provide a robustness check further below by changing $\eta$ from 0 to 1 .

We use the matching function $\mathcal{M}[\xi n, \xi(1-n)]=\xi n(1-n)$ with $\xi \in(0,2)$, and we choose $n=0.5$. This implies that the matching probabilities are $\delta=\delta^{p}=\xi / 2 \in(0,1)$. To identify a reasonable estimate for $\delta$, we need evidence of search frictions in the market for U.S. government securities. According to Duffie et al. (2005), search frictions in this type of market are mainly caused by time delays to contact counterparties. Pontrandolfo (2015) takes a deeper look at this issue and his methodology suggests that the probability of finding a suitable trading partner is lower than 1, especially for government bonds that were issued several years in the past. His methodology suggests that search frictions are significant, especially for market participants who would like to trade large quantities. ${ }^{28}$ In the light of this discussion, we set $\delta=0.8$ in the baseline calibration. Further below, we provide a robustness check by changing $\delta$ from 0 to 1 .

The parameters $\alpha$ and $A$, are obtained by matching the velocity of money and the elasticity of money demand simultaneously. We do this by minimizing the sum of squared differences between the target values and the respective model-generated moments. Following this calibration strategy, we are able to hit both targets exactly. The average velocity of money, measured as the ratio of GDP to M1, is equal to $v=6.30$ in the United States. ${ }^{29}$ The elasticity of money demand with respect to the long-term government bond yield, estimated by ordinary least squares and a $\log$ - $\log$ specification, is equal to $\varkappa=-0.21$.

The model's velocity of money is

$$
v=\frac{Y}{\phi M_{-1}}=\frac{1+(1-n)[\delta \hat{q}+(1-\delta) q]}{q},
$$

which depends on the interest rate in the primary bond market; i.e., $i \equiv 1 / \rho-1$, and on $\alpha$ via the functions $q$ and $\hat{q}{ }^{21}$ As for the empirical elasticity, the model's elasticity of money demand is estimated by ordinary least squares and a log-log specification.

The targets discussed above, and summarized in Table 1, are sufficient to calibrate all but one parameter: the taxation rate $\tau$. To address the question of whether it might be optimal to set $\tau>0$, we calibrate the model for $\tau=0$ and then calculate the optimal taxation rate $\tau^{*}$.

\footnotetext{
${ }^{27}$ This information has been communicated to us, but unfortunately we have no access to the trading data for that platform.

${ }^{28}$ We refer to Huber and Kim (2016) for a more detailed discussion about the trading frictions in financial markets.

${ }^{29}$ For the United States, we work with M1 adjusted for retail sweeps, instead of M1. Cynamon et al. (2006) show that the presence of commercial demand deposit sweep programs leads to an underreporting of transactions balances in M1. Furthermore, in Berentsen et al. (2015), it is shown that M1 adjusted for retail sweeps represents the available means of payments in the economy more accurately than M1. As the stock of money in the model is equal to the stock of means of payments, we map the model's money demand to the empirical money demand adjusted for retail sweeps.

${ }^{21}$ The model's velocity of money is derived as follows. The real output in the goods market is $Y_{G M}=$ $(1-n)[\delta \phi \hat{m}+(1-\delta) \phi m]$, where $\phi \hat{m}=\hat{q}$ and $\phi M_{-1}=\phi m=q$, and the real output in the primary bond market is $Y_{P B M}=1$ for $U(x)=\log (x)$. Accordingly, the total real output of the economy adds up to $Y=Y_{G M}+Y_{P B M}$, and the model-implied velocity of money is $v=Y / \phi M_{-1}$.
} 
Furthermore, we calculate $\Delta$, which is the percentage of total consumption that agents would be willing to give up in order to be in a steady state with $\tau=\tau^{*}$, instead of $\tau=0$. Finally, we are also interested in the share of market-2 consumption that is financed by the sale of bonds, defined as

$$
\kappa(\tau) \equiv \frac{\delta(\hat{q}-q)}{q+\delta(\hat{q}-q)} .
$$

In particular, we calculate the change in $\kappa$ associated to an increase in $\tau$; i.e., $\kappa\left(\tau^{*}\right) / \kappa(0)-1$.

\subsection{Baseline Results - United States}

Table 2 presents the results for the baseline calibration and three robustness checks. The robustness checks are defined as follows: in the calibration labeled "elasticity", we target an elasticity of money demand of -0.25 , instead of -0.21 ; in the calibration labeled "velocity", we target a velocity of money demand of 5.0, instead of 6.30 ; and in the calibration labeled "real rate", we reduce the real interest rate from 0.021 to 0.01 .

Table 2: Calibration Results for the United States ${ }^{\mathrm{a}}$

\begin{tabular}{llllll}
\hline \hline & Description & Baseline & Elasticity & Velocity & Real Rate \\
\hline$A$ & utility weight & 0.46 & 0.53 & 0.53 & 0.44 \\
$\alpha$ & relative risk aversion & 0.53 & 0.45 & 0.53 & 0.53 \\
$\tau^{*}$ & optimal tax & 0.016 & 0.016 & 0.016 & 0.014 \\
$\Delta$ & consumption delta & $0.002 \%$ & $0.002 \%$ & $0.003 \%$ & $0.001 \%$ \\
$s_{G M}$ & goods market size & 0.099 & 0.103 & 0.124 & 0.094 \\
$\frac{\kappa\left(\tau^{*}\right)}{\kappa(0)}-1$ & decrease in volume & -0.17 & -0.17 & -0.17 & -0.19 \\
$\gamma_{1}\left(\tau^{*}\right)$ & critical value & 0.988 & 0.988 & 0.988 & 0.997 \\
$\gamma_{2}\left(\tau^{*}\right)$ & critical value & 1.119 & 1.096 & 1.119 & 1.129 \\
$\overline{\mathcal{B}}\left(\tau^{*}\right)$ & critical value & 0.21 & 0.30 & 0.25 & 0.18 \\
\hline
\end{tabular}

${ }^{\text {a }}$ Table 2 displays the calibrated values for the key parameters $(A, \alpha)$, the optimal value for the tax, $\tau^{*}$, and the size of the goods market, $s_{G M}$. It also shows the percentage of total consumption, $\Delta$, that agents would be willing to give up in order to be in a steady state with $\tau=\tau^{*}$, instead of $\tau=0$. The table also displays the change in the share of market-2 consumption that is financed by the sale of bonds, the two critical inflation rates, and the critical bond-to-money ratio.

${ }^{\mathrm{b}}$ The optimal $\operatorname{tax} \tau^{*}$ is calculated numerically by searching for the welfare-maximizing value of $\tau$, holding all other parameters at their calibrated values.

For all calibrations, the optimal taxation rate is about 1.6 percent. The gain in total consumption from being in a steady state with $\tau=\tau^{*}$, as opposed to $\tau=0$, is around 0.002 percent. The estimates of the model-implied goods market share, $s_{G M}$, is around 10 percent, which is in line with related studies. ${ }^{30}$ Table 2 also shows that levying a transaction tax decreases the share of market-2 consumption that is financed by the sale of bonds. In particular, for all calibrations, $\kappa$ decreases by around 17 percent.

\footnotetext{
${ }^{30}$ See for instance Aruoba et al. (2011) and Lagos and Wright (2005).
} 
Table 2 also reports the critical values $\gamma_{1}\left(\tau^{*}\right)$ and $\gamma_{2}\left(\tau^{*}\right)$. To derive these values, we first calculate the optimal tax rate $\tau^{*}$ when $\gamma$ is set equal to the target. Then, using $\tau^{*}$, we can calculate $\gamma_{1}\left(\tau^{*}\right)$ and $\gamma_{2}\left(\tau^{*}\right)$ such that in the range $\gamma_{1}\left(\tau^{*}\right)<\gamma<\gamma_{2}\left(\tau^{*}\right)$ the type-I equilibrium exists. From the calibration results presented in Table 2, one can see that a higher elasticity results in a decrease of $\gamma_{2}\left(\tau^{*}\right)$, while changing the other targets has small effects.

To verify that only the type-I equilibrium is relevant for $\gamma_{1}\left(\tau^{*}\right)<\gamma<\gamma_{2}\left(\tau^{*}\right)$, the supply of bonds in the economy has to be sufficiently large. In the Supplementary Appendix, we derive conditions under which the bond constraint of the consumers binds. We find that it is not binding if $\mathcal{B}>\overline{\mathcal{B}}\left(\tau^{*}\right)$, where $\mathcal{B}$ is defined as the bond-to-money ratio, $B / M$, and

$$
\overline{\mathcal{B}}(\tau)=\frac{(1-\eta)[u(\hat{q})-u(q)]}{q}+\frac{\eta(\hat{q}-q)}{q(1-\tau)} .
$$

The quantities $\hat{q}$ and $q$ satisfy (20) and (21). In Table 2 , we report the value of $\overline{\mathcal{B}}\left(\tau^{*}\right)$. It is around 0.2 for all calibrations. In the data, we interpret the bond-to-money ratio as the ratio of the total public debt to the M1 money stock, and obtain an average value of $\mathcal{B}=4.3 .^{31}$ Thus, $\mathcal{B}>\overline{\mathcal{B}}\left(\tau^{*}\right)$ is satisfied, and we are in the type-I equilibrium. ${ }^{32}$

\subsection{The Role of Inflation}

We now study how the inflation rate $\gamma$ affects our results. For this purpose, we calculate the effect of increasing $\gamma$ on $\tau^{*}$ (Figure 3), and the effect of increasing $\gamma$ on $\Delta$ (Figure 4).

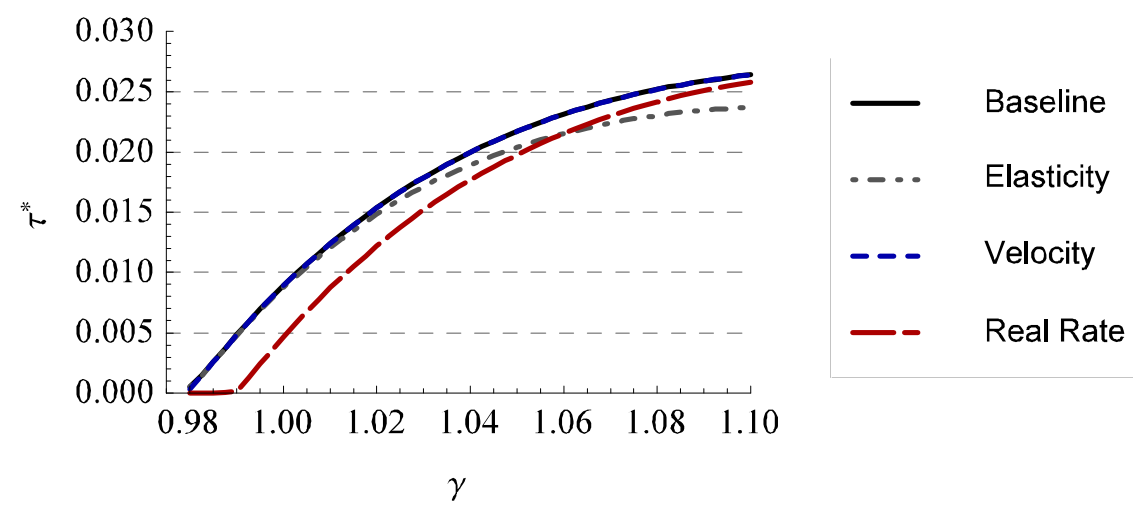

Figure 3: $\tau^{*}$ For inCREASing values of $\gamma$.

Figure 3 shows that $\tau^{*}$ is increasing in $\gamma$. Note, that the critical inflation rates depend on $\tau^{*}$ and, for each value of $\gamma$, we verify that we are in the type-I equilibrium. For the calibrations labeled

\footnotetext{
${ }^{31}$ This definition is in line with Berentsen et al. (2014) and Martin (2015).

${ }^{32}$ In contrast to the empirical study of Krishnamurthy and Vissing-Jorgensen (2012), our calibration does not support a liquidity premium for bonds.
} 
"elasticity" and "real rate", the type-I equilibrium exists for inflation rates up to 11 percent, while, for the other two calibrations, it exists for inflation rates of more than 14 percent.

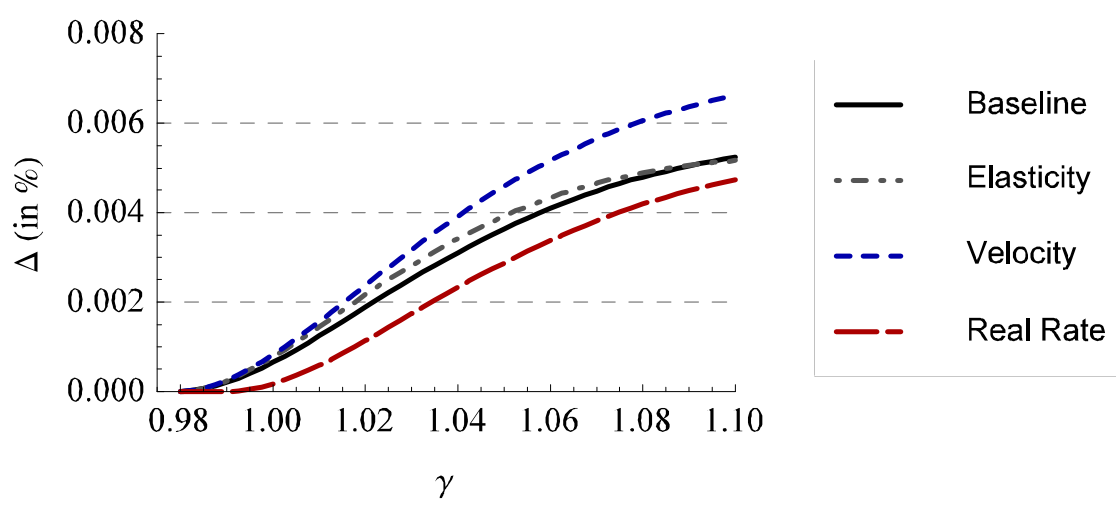

Figure 4: $\Delta$ FOR INCREASING VALUES OF $\gamma$.

Figure 4 shows that the benefit from being in a steady state with $\tau=\tau^{*}$, as opposed to $\tau=0$, is also increasing in $\gamma$. A lower velocity of money has the biggest impact on $\Delta$ for higher inflation rates and results in $\Delta \approx 0.007$ percent for $\gamma=1.10$.

\subsection{The Role of Search and Bargaining Frictions}

For the baseline calibration, we have set the matching probabilty to $\delta=0.8$ and the bargaining power of the consumer to $\eta=0.5$. In what follows, we calculate the optimal FTT for different values of these parameters.

Matching probability Figure 5 plots the change in $\tau^{*}$ as $\delta$ increases from 0 to 1 for $\eta=0.5$. Figure 6 plots how much consumption the representative consumer would be willing to sacrifice in order to be taxed at the optimal tax rate $\tau^{*}$ relative to $\tau=0 .{ }^{33}$

\footnotetext{
${ }^{33}$ In this paragraph, we estimate the model's interest rate elasticity of money demand using a point approximation at the calibrated value of $\gamma$. In Berentsen et al. (2015), it is shown that when estimating the model's elasticity of money demand by ordinary least squares and a log-log specification, the standard errors are small. Thus, a point approximation at the calibrated value of $\gamma$ represents a reasonable approximation for the model's elasticity of money demand.
} 


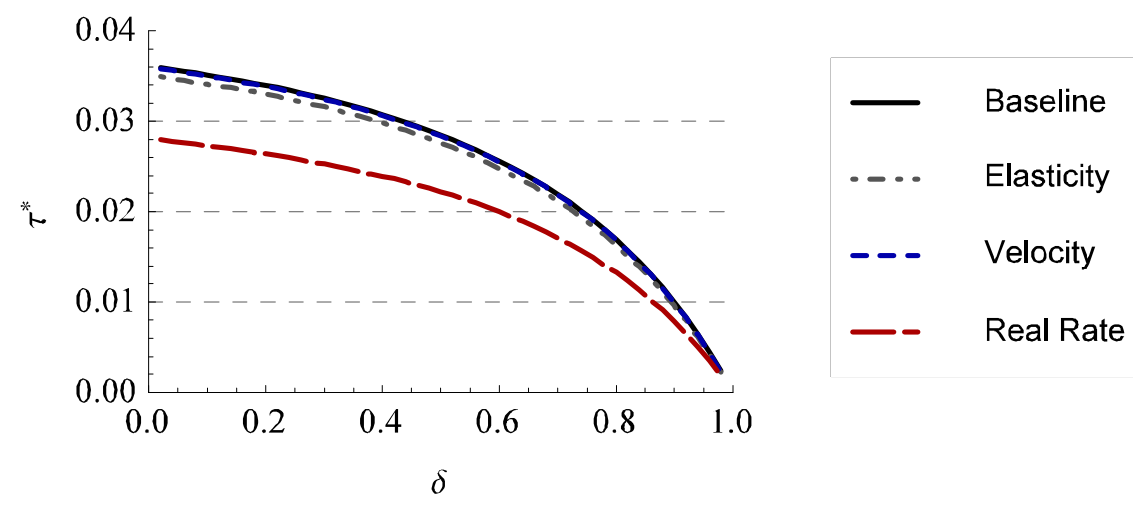

Figure 5: $\tau^{*}$ FOR INCREASING VAlUES OF $\delta$.

We find numerically that $\tau^{*}$ is decreasing in $\delta$. Note that $\tau^{*}$ approaches 0 for $\delta \rightarrow 1$ for all calibrations. The reason is that at $\delta=1$, all agents are active and so there is no role for a FTT (see Section 7 for further details).

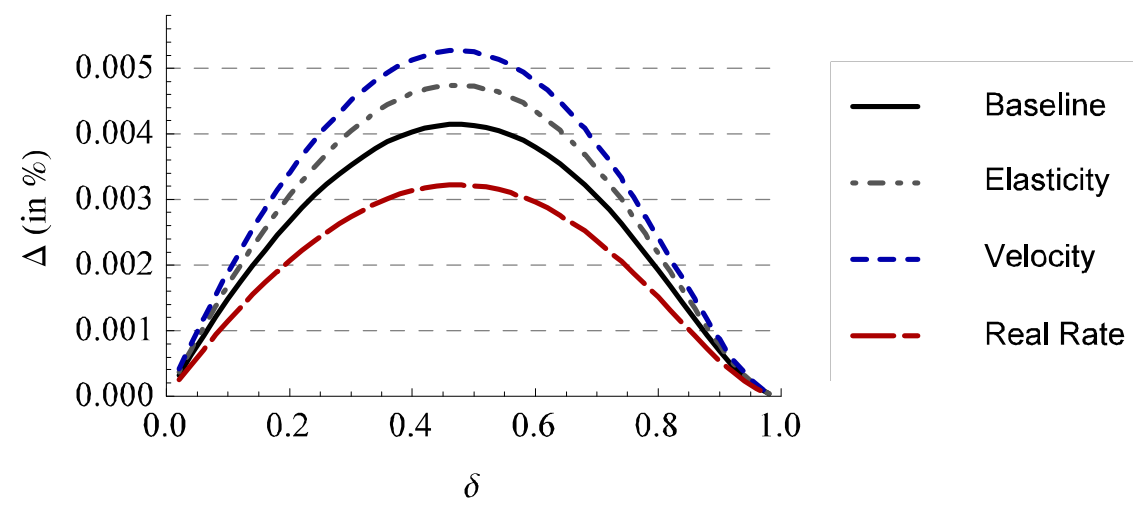

Figure 6: $\Delta$ FOR INCREASING VAlUES OF $\delta$.

Here, we also find that $\Delta$ approaches 0 for $\delta \rightarrow 1$ for all calibrations. Furthermore, $\Delta$ is maximized at $\delta=0.5$. At this value, the fraction of consumers who benefit from the tax (the passive agents) equals the fraction of consumers who are penalised (the active agents).

Bargaining power Figure 7 plots the change of $\tau^{*}$ as $\eta$ increases from 0 to 1 for $\delta=0.8$. Figure 8 plots how much consumption the representative consumer would be willing to sacrifice in order to be taxed at the optimal tax rate $\tau^{*}$ relative to $\tau=0$, for different values of $\eta$. 


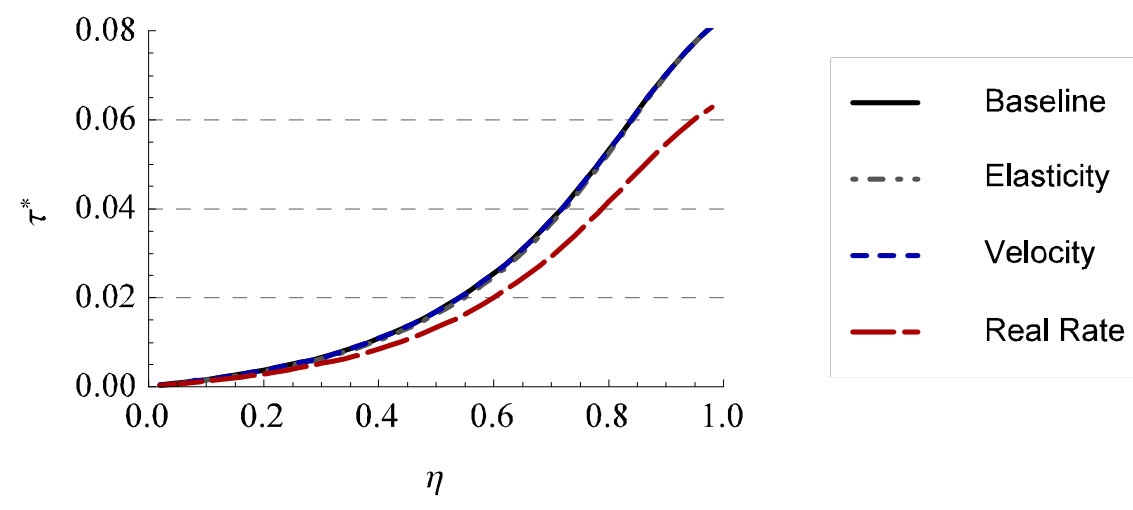

FiguRE 7: $\tau^{*}$ FOR INCREASING VALUES OF $\eta$.

We find numerically that the optimal taxation rate is increasing in $\eta$. In particular, $\tau^{*}$ increases to 8 percent for $\eta \rightarrow 1$ for the baseline calibration. For higher values of $\eta$, active consumers obtain a larger fraction of the trade surplus in the secondary bond market and, consequently, their demand for money decreases in $\eta$. Thus, the optimal taxation rate is higher to induce agents to demand more money.

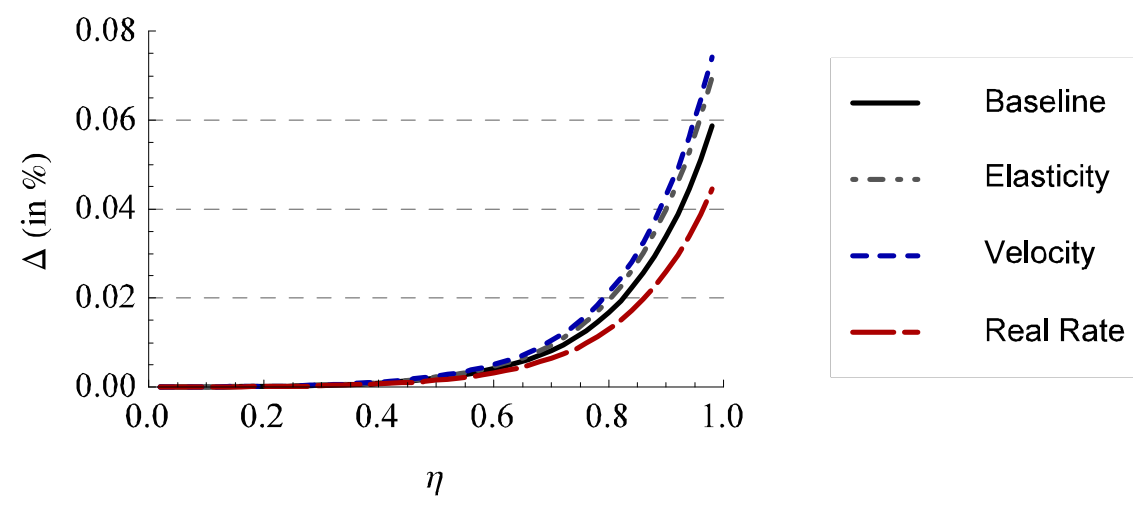

Figure 8: $\Delta$ FOR INCREASING VALUES OF $\eta$.

The benefit of the optimal tax as measured by $\Delta$ is also increasing in $\eta$. We obtain $\Delta \approx 0.059$ percent for $\eta \rightarrow 1$ for the baseline calibration. Thus, the higher the bargaining power of consumers in the secondary bond market, the higher the optimal FTT and also the benefits in terms of welfare. 


\subsection{Germany}

As a robustness exercise, we now calibrate our model to Germany. The velocity of money in Germany $(v=0.70)$ is substantially lower than that in the United States $(v=6.3)$. The elasticity of money demand is similar in both countries. Furthermore, in Germany, the average inflation rate is nearly 1 percent lower, and the real interest rate is 0.5 percent higher than for the United States. As for the United States, we assume $\delta=0.8$ and $\eta=0.5$. Table 3 summarizes the calibration targets.

Table 3: Calibration targets for Germany

\begin{tabular}{ll}
\hline \hline Target Description & Germany \\
\hline Average real interest rate & 0.026 \\
Average inflation rate & 0.015 \\
Average velocity of money & 0.70 \\
Average elasticity of money demand & $-0.21(0.079)$ \\
\hline Standard errors in parentheses. &
\end{tabular}

As we did for the United States, we calibrate the model to the targets presented in Table 3 and perform three robustness checks, for which we use exactly the same targets as for the United States. ${ }^{34}$ Table 4 shows the calibration results for Germany.

TABle 4: Results For Germany ${ }^{\mathrm{a}}$

\begin{tabular}{llllll}
\hline \hline & Description & Baseline & Elasticity & Velocity & Real Rate \\
\hline$A$ & utility weight & 2.82 & 2.74 & 0.56 & 2.26 \\
$\alpha$ & relative risk aversion & 0.42 & 0.35 & 0.48 & 0.42 \\
$\tau^{*}$ & optimal $\tau^{\mathrm{b}}$ & 0.015 & 0.014 & 0.015 & 0.010 \\
$\Delta$ & consumption delta & $0.018 \%$ & $0.021 \%$ & $0.003 \%$ & $0.008 \%$ \\
$s_{G M}$ & goods market size & 0.848 & 0.893 & 0.125 & 0.771 \\
$\frac{\kappa\left(\tau^{*}\right)}{\kappa(0)}-1$ & decrease in volume & -0.17 & -0.16 & -0.18 & -0.20 \\
$\gamma_{1}\left(\tau^{*}\right)$ & critical value & 0.982 & 0.982 & 0.983 & 0.995 \\
$\gamma_{2}\left(\tau^{*}\right)$ & critical value & 1.081 & 1.063 & 1.098 & 1.096 \\
$\overline{\mathcal{B}}\left(\tau^{*}\right)$ & critical value & 0.30 & 0.37 & 0.25 & 0.17 \\
\hline
\end{tabular}

${ }^{a}$ Table 4 is Table 2's counterpart for Germany. For a description of the reported variables, we refer the reader to Table

2 .

Table 4 shows that, for all but one calibration, the goods market share is around 85 percent in Germany. As a consequence, there is a significantly higher benefit from being in a steady state with $\tau=\tau^{*}$ as opposed to $\tau=0$. For example, in the baseline calibration, we obtain $\Delta$ $\approx 0.018$ percent, which is around ten times higher than that for the United States. The main

\footnotetext{
${ }^{34}$ That is, in the calibration labeled "elasticity", we target an elasticity of money demand of -0.25 ; in the calibration labeled "velocity", we target a velocity of money demand of 5.0; and in the calibration labeled "real rate", we target a real interest rate of 0.01 .
} 
reason behind this result is the lower velocity of money, which becomes clear when we look at the calibration labeled "velocity". When we increase the target of the velocity from $v=0.70$ to $v=5.0$, the benefit of raising the tax from zero to $\tau=\tau^{*}$ drops to $\Delta \approx 0.003$ percent. Similar to the United States, the share of market-2 consumption that is financed by the sale of bonds decreases by around 17 percent when $\tau=\tau^{*}$ as compared to $\tau=0$. Furthermore, the optimal taxation rate is around 1.5 percent, which is close to the estimate that we obtain for the United States. This is mainly because we assume the same financial market parameters for both countries; i.e., $\delta=0.8$ and $\eta=0.5$. Empirically, we obtain for Germany an average bond-to-money ratio equaling $\mathcal{B}=0.50$, and hence, for all calibrations presented in Table 4 , we have $\mathcal{B}>\overline{\mathcal{B}}\left(\tau^{*}\right)$ at the calibrated value of $\gamma$.

\section{Conclusion}

We provide a novel justification for imposing a financial transaction tax (FTT). We develop the argument in a monetary model where agents hold money to finance stochastic consumption opportunities. Away from the Friedman rule, the cost of holding money is positive, and in order to mitigate this inflation tax, agents can store their wealth in a nominal interest-bearing bond instead; this bond cannot be used to pay for consumption, but it can be sold in a OTC secondary bond market to obtain money when needed.

We show that the availability of this mitigation instrument is not necessarily good for welfare. On the one hand, agents who liquidate their bonds are able to buy more consumption ex-post. On the other hand, all agents anticipate this and therefore hold less money ex-ante, which means they will obtain even less consumption in the event that they fail to make a trade in the secondary bond market. We show that a FTT paid by traders in the secondary bond market (proportional to the size of the trade) will make it more costly to rely on the secondary bond market: Therefore, agents will carry more money ex-ante and may still be able to attain a high level of consumption ex-post. The FTT can therefore improve welfare in a second-best sense, and it has the advantage over the (first-best) Friedman rule that it does not require taxes in order to be implemented but, on the contrary, raises revenue.

We calibrate our model using U.S. and German data, and for both countries we find that a FTT of about 1.6 percent is constrained-optimal. The optimal tax rate reduces the real volume of financial trading by 17 percent. Furthermore, we show that the optimal FTT is decreasing in the matching probability and consequently the optimal FTT is lower for markets with smaller search frictions. 


\section{Appendix}

Derivation of $\Delta^{c}$ and $\Delta^{p}$. They are defined as follows:

$$
\begin{aligned}
\Delta^{c} & \equiv V_{2}^{c}\left[m+d_{m}(1-\tau), b-d_{b}\right]-V_{2}^{c}(m, b), \\
\Delta^{p} & \equiv V_{2}^{p}\left(m-d_{m}, b+d_{b}\right)-V_{2}^{p}(m, b) .
\end{aligned}
$$

Let us start with $\Delta^{c}$ and write it as follows,

$$
\Delta^{c}=u\left(\hat{q}_{c}\right)+V_{3}\left[m+d_{m}(1-\tau)-p \hat{q}_{c}, b-d_{b}\right]-\left[u\left(q_{c}\right)+V_{3}\left(m-p q_{c}, b\right)\right] .
$$

The consumer's surplus, $\Delta^{c}$, is given by his payoff from trading bonds for money in a match in the secondary bond market, $u\left(\hat{q}_{c}\right)+V_{3}\left[m+d_{m}(1-\tau)-p \hat{q}_{c}, b-d_{b}\right]$, minus his payoff from not trading, $u(q)+V_{3}\left(m-p q_{c}, b\right)$.

If a trade takes place, the consumer receives $d_{m}(1-\tau)$ units of money for $d_{b}$ units of bonds, and he spends $p \hat{q}_{c}=m+d_{m}(1-\tau)$ units of money and consumes $\hat{q}_{c}$ units of goods. If no trade takes place in the secondary bond market, the consumer spends $p q_{c}=m$ units of money and consumes $q_{c}$ units of goods. Simplifying and rearranging terms, the consumer's net surplus $\Delta^{c}$ can be rewritten as

$$
\Delta^{c}=u\left(\hat{q}_{c}\right)-u\left(q_{c}\right)-\phi d_{b},
$$

where we have used $p \hat{q}_{c}=p q_{c}+d_{m}(1-\tau)$, the consumer's value function in the goods market, $V_{2}^{c}$, and the linearity of $V_{3}$.

Let us now derive $\Delta^{p}$ and write

$$
\Delta^{p}=-c\left(q_{c}\right)+V_{3}\left(m-d_{m}+p q_{c}, b+d_{b}\right)-\left[-c\left(q_{c}\right)+V_{3}\left(m+p q_{c}, b\right)\right] .
$$

The producer's surplus, $\Delta^{p}$, is given by her payoff from trading bonds for money in a match in the secondary bond market minus her payoff from not trading. Note that the quantity of goods produced by a producer in the goods market is not affected by what happens in the secondary bond market. This is because the produced quantity depends on the consumer's money holdings, not the producer's money holdings. Hence, the producer's surplus is $-c\left(q_{c}\right)+$ $V_{3}\left(m-d_{m}+p q_{c}, b+d_{b}\right)$ if she trades in the secondary bond market, while it is $-c\left(q_{c}\right)+V_{3}\left(m+p q_{c}, b\right)$ if she does not.

Rearranging and simplifying terms, we obtain

$$
\Delta^{p}=\phi d_{b}-\phi d_{m}
$$

Note that this constraint implies that the interest rate in the secondary bond market is nonnegative, since the interest rate is $\frac{d_{b}}{d_{m}}-1$.

Proof of Proposition 1. Derivation of (20). In a type-I equilibrium, both the producer's cash constraint and the consumer's bond constraint are nonbinding in the secondary bond market (i.e., $\lambda^{p}=\lambda^{c}=0$ ). In this case, the first-order condition in the secondary bond market (19) reduces to $0=(1-\tau) u^{\prime}(\hat{q})-1$. 
Derivation of (21). In a type-I equilibrium, the marginal value of money of an agent entering the secondary bond market, before the realization of the idiosyncratic shock, is

$$
\frac{\partial V_{1}(m, b)}{\partial m}=(1-n) \frac{\partial V_{1}^{c}(m, b)}{\partial m}+n \frac{\partial V_{1}^{p}(m, b)}{\partial m} .
$$

In the Supplementary Appendix, we show that

$$
\begin{aligned}
& \frac{\partial V_{1}^{c}(m, b)}{\partial m}=\phi\left\{\delta\left[(1-\eta) u^{\prime}(q)+\eta u^{\prime}(\hat{q})\right]+(1-\delta) u^{\prime}(q)\right\} \\
& \frac{\partial V_{1}^{p}(m, b)}{\partial m}=\phi .
\end{aligned}
$$

Using (4) updated one period, we obtain (21).

Derivation of (22). In a type-I equilibrium, the marginal value of bonds of an agent entering the secondary bond market, before the realization of the idiosyncratic shock, is

$$
\frac{\partial V_{1}(m, b)}{\partial b}=(1-n) \frac{\partial V_{1}^{c}(m, b)}{\partial b}+n \frac{\partial V_{1}^{p}(m, b)}{\partial b} .
$$

In the Supplementary Appendix, we show that $(1-n) \frac{\partial V_{1}^{c}(m, b)}{\partial b}+n \frac{\partial V_{1}^{p}(m, b)}{\partial b}=\phi$. Using (4) updated one period, we get (22).

Proof of Proposition 2. Derivation of (23). In a type-II equilibrium, a producer is cashconstrained in the secondary bond market, thus $d_{m}=m=M$. Also note that $m+(1-\tau) d_{m}=p \hat{q}$ and $m=p q$. Eliminate $m$ in the former equation using the latter, then use $d_{m}=m$, and rearrange terms to obtain (23).

Derivation of (24). The marginal value of money of an agent entering the secondary bond market, before the realization of the idiosyncratic shock, can be written as follows:

$$
\frac{\partial V_{1}(m, b)}{\partial m}=(1-n) \frac{\partial V_{1}^{c}(m, b)}{\partial m}+n \frac{\partial V_{1}^{p}(m, b)}{\partial m} .
$$

In the Supplementary Appendix, we show that

$$
\begin{aligned}
& \frac{\partial V_{1}^{c}(m, b)}{\partial m}=\phi\left\{\delta\left[(1-\eta) u^{\prime}(q)+\eta u^{\prime}(\hat{q})\right]+(1-\delta) u^{\prime}(q)\right\}, \\
& \frac{\partial V_{1}^{p}(m, b)}{\partial m}=\phi\left\{\delta^{p}\left[(1-\eta)(1-\tau) u^{\prime}(\hat{q})+\eta\right]+1-\delta^{p}\right\} .
\end{aligned}
$$

Using (4) updated one period, we obtain (24).

Derivation of (25). In a type-II equilibrium, the marginal value of bonds of an agent entering the secondary bond market, before the realization of the idiosyncratic shock, is

$$
\frac{\partial V_{1}(m, b)}{\partial b}=(1-n) \frac{\partial V_{1}^{c}(m, b)}{\partial b}+n \frac{\partial V_{1}^{p}(m, b)}{\partial b} .
$$

In the Supplementary Appendix, we show that $(1-n) \frac{\partial V_{1}^{c}(m, b)}{\partial b}+n \frac{\partial V_{1}^{p}(m, b)}{\partial b}=\phi$. Using (4) updated one period, we obtain (25). 
Proof of Proposition 3. The critical value $\gamma_{1}$ is the value of $\gamma$ such that $\hat{q}=q$ in Proposition 1. If $\hat{q}=q$, from (20) and (21) we have

$$
\gamma_{1}=\beta \frac{1-n \tau}{1-\tau}
$$

Since $u^{\prime}(q)$ is strictly decreasing in $q, \gamma_{1}$ is unique. Note that for $\tau=0, \gamma_{1}=\beta$ and for $\tau>0$, we have $\gamma_{1}>\beta$.

The critical value $\gamma_{2}$ is the value of $\gamma$ such that the quantities $q$ and $\hat{q}$ in Proposition 2 are equal to the respective quantities $q$ and $\hat{q}$ in Proposition 1. To that end, from (20), we have $u^{\prime}(\hat{q})(1-\tau)=1$, which can be solved for $\hat{q}(\tau)=u^{\prime-1}\left[(1-\tau)^{-1}\right]$. Next, from (23) we have $q=\hat{q}(2-\tau)^{-1}$. Use the previous expression to replace $\hat{q}$ to obtain $q(\tau)=\hat{q}(\tau)(2-\tau)^{-1}$. Finally, use these expressions in (21) or (24) to obtain

$$
\gamma_{2}=\beta\left\{(1-n)\left\{\delta\left[(1-\eta) u^{\prime}(q(\tau))+\frac{\eta}{1-\tau}\right]+(1-\delta) u^{\prime}(q(\tau))\right\}+n\right\} .
$$

Note that $\gamma_{1} \leq \gamma_{2}$ if $1 \leq(1-\tau)[1-\eta \delta] u^{\prime}(q(\tau))+\eta \delta$, which requires that the tax is not too high.

Proof of Proposition 4. Use $\frac{d \hat{q}}{d \tau}=\frac{1}{(1-\tau)^{2} u^{\prime \prime}(\hat{q})}$ and $\frac{d q}{d \tau}=-\frac{\delta \eta}{(1-\delta \eta)(1-\tau)^{2} u^{\prime \prime}(q)}$ to replace $\frac{d \hat{q}}{d \tau}$ and $\frac{d q}{d \tau}$ in (27) and rearrange the resulting expression to get

$$
\frac{1-\beta}{(1-n)} \frac{d \mathcal{W}}{d \tau}=\delta\left[u^{\prime}(\hat{q})-1\right] \frac{1}{(1-\tau)^{2} u^{\prime \prime}(\hat{q})}\left\{1-\frac{(1-\delta)\left[u^{\prime}(q)-1\right]}{\delta\left[u^{\prime}(\hat{q})-1\right]} \frac{\delta \eta u^{\prime \prime}(\hat{q})}{(1-\delta \eta) u^{\prime \prime}(q)}\right\} .
$$

Since $\delta\left[u^{\prime}(\hat{q})-1\right] \frac{1}{(1-\tau)^{2} u^{\prime \prime}(\hat{q})}<0, \frac{d \mathcal{W}}{d \tau}>0$ requires that the term in curly brackets is negative. This is the case if $(28)$ holds.

Data Source. The data we use for the calibration is downloadable from the St. Louis FRED ${ }^{\circledR}$ database, except for the consumer price index of Germany, which we downloaded from Bloomberg. Table A.1 gives a brief overview of the data sources for the United States and Germany.

Table A.1: Data source

\begin{tabular}{lllll}
\hline \hline Country & Description & Identifier & Period & Frequency \\
\hline U.S. & Long-term gov. bond yield & IRLTLT01USM156N & 95:Q1-13:Q4 & quarterly \\
U.S. & M1 adj. for retail sweeps & M1ADJ & 95:Q1-13:Q4 & quarterly \\
U.S. & Nominal GDP & GDP & 95:Q1-13:Q4 & quarterly \\
U.S. & Consumer price index & CPIAUCSL & 95:Q1-13:Q4 & quarterly \\
U.S. & Total public debt & GFDEBTN & 95:Q1-13:Q4 & quarterly \\
\hline Germany & Long-term gov. bond yield & IRLTLT01DEM156N & 95:Q1-13:Q4 & quarterly \\
Germany & M1 & MYAGM1DEM189S & 95:Q1-13:Q4 & quarterly \\
Germany & Nominal GDP & DEUGDPNQDSMEI & 95:Q1-13:Q4 & quarterly \\
Germany & Consumer price index & GRCP20YY Index & 95:Q1-13:Q4 & quarterly \\
Germany & Total public debt & GGGDTADEA188N & 95:Q1-13:Q4 & yearly \\
\hline
\end{tabular}


For the consumer price index of Germany, we applied the same aggregation method to calculate the quarterly values as used by the St. Louis FRED ${ }^{\circledR}$ database, which is defined as the average of the monthly data. For the total public debt of Germany, which is only available at a yearly frequency, we assumed that the debt level remains unchanged within a year. 


\section{References}

[1] Andolfatto, D., 2010, "Essential Interest-Bearing Money," Journal of Economic Theory, 145, 1319-1602.

[2] Andolfatto, D., 2013, "Incentive-Feasible Deflation," Journal of Monetary Economics, 60, 383-390.

[3] Aruoba, S. B., Waller, C., and Wright, R., 2011, "Money and Capital," Journal of Monetary Economics, 58, 98-116.

[4] Aruoba, B., Rocheteau, G., and Waller, C., 2007, "Bargaining and the Value of Money," Journal of Monetary Economics, 54, 2636-2655.

[5] Berentsen, A., and Monnet, C., 2008, "Monetary Policy in a Channel System," Journal of Monetary Economics, 55, 1067-1080.

[6] Berentsen, A., Camera, G., and Waller, C., 2007, "Money, Credit and Banking," Journal of Economic Theory, 135, 171-195.

[7] Berentsen, A., Huber, S., and Marchesiani, A., 2014, "Degreasing the Wheels of Finance," International Economic Review, 55, 735-763.

[8] Berentsen, A., Huber, S., and Marchesiani, A., 2015, "Financial Innovations, Money Demand, and the Welfare Cost of Inflation," Journal of Money, Credit, and Banking, 47, 223-261.

[9] Berentsen, A., Huber, S., and Marchesiani, A., 2016, "Limited Commitment and the Demand for Money," University of Zurich, working paper.

[10] Berentsen, A., and Waller, C., 2011, "Outside Versus Inside Bonds: A Modigliani-Miller Type Result for Liquidity Constrained Economies," Journal of Economic Theory, 146, 18521887.

[11] Bianchi, J., and Mendoza, E. G., 2011, "Overborrowing, Financial Crises and 'Macroprudential' Policy," International Monetary Fund, working paper.

[12] Caballero, R. J., and Krishnamurthy, A., 2003, "Excessive Dollar Debt: Financial Development and Underinsurance," Journal of Finance, 58, 867-894.

[13] Chiu, J., Dong, M., and Shao, E., 2012, "On the Welfare Effects of Credit Arrangements," Bank of Canada, working paper.

[14] Chiu, J., Meh, C., and Wright, R., 2016, "Innovation and Growth with Financial, and other, Frictions," International Economic Review, forthcoming. 
[15] Chiu, J., and Monnet, C., 2014, "Relationship Lending in a Tiered Interbank Market," University of Bern, working paper.

[16] Cynamon, B. Z., Dutkowsky, D. H., and Jones, B. E., 2006, "Redefining the Monetary Aggregates: A Clean Sweep." Eastern Economic Journal, 32, 661-673.

[17] Dávila, E., 2015, "Optimal Financial Transaction Taxes," New York University, working paper.

[18] Dow, J., and Rahi, R., 2000, "Should Speculators Be Taxed?" Journal of Business, 73, 89-107.

[19] Duffie, D., Gârleanu, N., and Pedersen, L. H., 2005, "Over-the-Counter Markets," Econometrica, 73, 1815-1847.

[20] Geromichalos, A., and Herrenbrueck, L., 2016, "Monetary Policy, Asset Prices, and Liquidity in Over-the-Counter Markets," Journal of Money, Credit and Banking, 48, 35-79.

[21] Geromichalos, A., Herrenbrueck, L., and Salyer, K. D., 2016, "A Search-Theoretic Model of the Term Premium," Theoretical Economics, forthcoming.

[22] Greenwald, B., and Stiglitz J., 1986, "Externalities in Economies with Imperfect Information and Incomplete Markets," Quarterly Journal of Economics, 101, 229-264.

[23] Gu, C., Mattesini, F., Monnet, C., and Wright, R., 2013a, "Banking: A New Monetarist Approach," Review of Economic Studies, 80, 636-662.

[24] Gu, C., Mattesini, F., Monnet, C., and Wright, R., 2013b, "Endogenous Credit Cycles," Journal of Political Economy, 121, 940-965.

[25] Huber, S., and Kim, J., 2016, "Central Bank Purchases of Government Bonds" University of Zurich, working paper.

[26] Jeanne, O., and Korinek, A., 2012, "Managing Credit Booms and Busts: A Pigouvian Taxation Approach", NBER, working paper.

[27] Kalai, E., 1977, "Proportional Solutions to Bargaining Situations: Interpersonal Utility Comparisons," Econometrica, 7, 1623-1630.

[28] Kiyotaki, N., and Wright, R., 1989, "On Money as a Medium of Exchange," Journal of Political Economy, 97, 927-954.

[29] Kocherlakota, N., 1998, "Money is Memory," Journal of Economic Theory, 81, 232-251.

[30] Kocherlakota, N., 2003, "Societal Benefits of Illiquid Bonds," Journal of Economic Theory, 108, 179-193. 
[31] Korinek, A., 2012, "Systemic Risk-Taking: Amplification Effects, Externalities, and Regulatory Responses," University of Maryland, working paper.

[32] Krishnamurthy, A., and Vissing-Jorgensen, A., 2012, "The Aggregate Demand for Treasury Debt," Journal of Political Economy, 120, 233-267.

[33] Kyle, A. S., 1985 "Continuous Auctions and Insider Trading," Econometrica, 53, 1315-1335.

[34] Lagos, R., and Rocheteau, G., 2009, "Liquidity in Asset Markets with Search Frictions," Econometrica, 77, 403-426.

[35] Lagos, R., Rocheteau, G., and Weill, P. O., 2011, "Crises and Liquidity in Over-the-Counter Markets," Journal of Economic Theory, 146, 2169-2205.

[36] Lagos, R., and Wright, R., 2005, "A Unified Framework for Monetary Theory and Policy Evaluation," Journal of Political Economy, 113, 463-484.

[37] Lagos, R., and Zhang S., 2015, "Monetary Exchange in Over-The-Counter Markets: A Theory of Speculative Bubbles, the Fed Model, and Self-fulfilling Liquidity Crises," NBER, working paper.

[38] Li, Y., 2011, "Currency and Checking Deposits as Means of Payment," Review of Economic Dynamics, 14, 403-417.

[39] Li, Y., and Li, Y. S., 2013, "Liquidity and Asset Prices: A New Monetarist Approach," Journal of Monetary Economics, 60, 426-438.

[40] Li, Y., Rocheteau, G., and Weill, P. O., 2012, "Liquidity and the Threat of Fraudulent Assets," Journal of Political Economy, 120, 815-846.

[41] Lorenzoni, G., 2008, "Inefficient Credit Booms," Review of Economic Studies, 75, 809-833.

[42] Martin, F., 2015, "Debt, Inflation and Central Bank Independence," European Economic Review, 79,129-150.

[43] Mattesini, F., and Nosal, E., 2016, "Liquidity and Asset Prices in a Monetary Model with OTC Asset Markets," Journal of Economic Theory, 164, 187-217.

[44] Moore, J., 2013, "Pecuniary Externality through Credit Constraints: Two Examples without Uncertainty," University of Edinburgh, working paper.

[45] Pontrandolfo, F., 2015, "LQA Liquidity Assessment Tool", Bloomberg L.P.

[46] Pomeranets, A., 2012, "Financial Transaction Taxes: International Experiences, Issues and Feasibility," Bank of Canada Review, Autumn.

[47] Rocheteau, G., and Wright, R., 2005, "Money in Competitive Equilibrium, in Search Equilibrium, and in Competitive Search Equilibrium," Econometrica, 73, 175-202. 
[48] Rocheteau, G., and Wright, R., 2013, "Liquidity and Asset Market Dynamics," Journal of Monetary Economics, 60, 275-294.

[49] Sanches, D., and Williamson, S., 2010, "Money and Credit with Limited Commitment and Theft," Journal of Economic Theory, 145, 1525-1549.

[50] Subrahmanyam, A., 1998, "Transaction Taxes and Financial Market Equilibrium," Journal of Business, 71, 81-118.

[51] Tobin, J., 1978, "A Proposal for International Monetary Reform," Eastern Economic Journal, 4, 153-159.

[52] Wallace, N., 2001, "Whither Monetary Economics?" International Economic Review, 42, 847-869.

[53] Williamson, S., 2012, "Liquidity, Monetary Policy, and the Financial Crisis: A New Monetarist Approach." American Economic Review, 102, 2570-2605.

[54] Williamson S., and Wright R., 2010a, "New Monetarist Economics: Methods," Federal Reserve Bank of St. Louis Review, May, 265-302.

[55] Williamson, S., and Wright, R., 2010b, "New Monetarist Economics: Models," Handbook of Monetary Economics, in: Benjamin M. Friedman \& Michael Woodford (ed.), Handbook of Monetary Economics, edition 1, volume 3, chapter 2, 25-96. 